\title{
ABUNDANT MOLECULAR GAS AND INEFFICIENT STAR FORMATION IN INTRACLUSTER REGIONS: RAM PRESSURE STRIPPED TAIL OF THE NORMA GALAXY ESO137-001*
}

\author{
Pavel Jáchym ${ }^{1}$, Françoise Combes ${ }^{2}$, Luca Cortese ${ }^{3}$, Ming Sun ${ }^{4}$, and Jefreey D. P. Kenney ${ }^{5}$ \\ ${ }^{1}$ Astronomical Institute, Academy of Sciences of the Czech Republic, Boční II 1401, 14100 Prague, Czech Republic; jachym@ig.cas.cz \\ ${ }^{2}$ Observatoire de Paris, LERMA, 61 Av. de l'Observatoire, F-75014 Paris, France \\ ${ }^{3}$ Centre for Astrophysics \& Supercomputing, Swinburne University of Technology, Mail H30, P.O. Box 218, Hawthorn, VIC 3122, Australia \\ ${ }^{4}$ Department of Physics, University of Alabama in Huntsville, 301 Sparkman Drive, Huntsville, AL 35899, USA \\ ${ }^{5}$ Department of Astronomy, Yale University, 260 Whitney Avenue, New Haven, CT 06511, USA \\ Received 2014 March 10; accepted 2014 June 27; published 2014 August 8
}

\begin{abstract}
For the first time, we reveal large amounts of cold molecular gas in a ram-pressure-stripped tail, out to a large "intracluster" distance from the galaxy. With the Actama Pathfinder EXperiment (APEX) telescope, we have detected ${ }^{12} \mathrm{CO}(2-1)$ emission corresponding to more than $10^{9} M_{\odot}$ of $\mathrm{H}_{2}$ in three $\mathrm{H} \alpha$ bright regions along the tail of the Norma cluster galaxy ESO 137-001, out to a projected distance of $40 \mathrm{kpc}$ from the disk. ESO 137-001 has an $80 \mathrm{kpc}$ long and bright X-ray tail associated with a shorter $(40 \mathrm{kpc})$ and broader tail of numerous star forming $\mathrm{H} \mathrm{II}$ regions. The amount of $\sim 1.5 \times 10^{8} \mathrm{M}_{\odot}$ of $\mathrm{H}_{2}$ found in the most distant region is similar to molecular masses of tidal dwarf galaxies, though the standard Galactic CO-to- $\mathrm{H}_{2}$ factor could overestimate the $\mathrm{H}_{2}$ content. Along the tail, we find the amount of molecular gas to drop, while masses of the X-ray-emitting and diffuse ionized components stay roughly constant. Moreover, the amounts of hot and cold gas are large and similar, and together nearly account for the missing gas from the disk. We find a very low SFE $\left(\tau_{\text {dep }}>10^{10} \mathrm{yr}\right)$ in the stripped gas in ESO 137-001 and suggest that this is due to a low average gas density in the tail, or turbulent heating of the interstellar medium that is induced by a ram pressure shock. The unprecedented bulk of observed $\mathrm{H}_{2}$ in the ESO 137-001 tail suggests that some stripped gas may survive ram pressure stripping in the molecular phase.
\end{abstract}

Key words: galaxies: clusters: individual (A3627) - galaxies: evolution - galaxies: individual (ESO 137-001) galaxies: ISM - galaxies: star formation - submillimeter: ISM

Online-only material: color figures

\section{INTRODUCTION}

The dense environments of galaxy clusters and groups have been identified as places where transformations of galaxies from blue star-forming to red gas-poor systems occur. Latetype galaxies are rare in the cores of galaxy clusters, although they dominate in their outer parts and outside of clusters (the morphology-density relation; Dressler 1980; van der Wel et al. 2010). Cluster galaxy populations evolve over cosmic time, as shown by the excess of optically blue galaxies in clusters at higher redshifts (the Butcher-Oemler effect; Butcher \& Oemler 1978, 1984). Several mechanisms are active in clusters that could account for the observed evolution, such as mutual gravitational interactions of galaxies, including mergers and rapid galaxy encounters, alias harassment, the tidal influence of the cluster potential, and ram pressure of the intracluster medium (ICM) on the interstellar matter (ISM) of member galaxies (Gunn \& Gott 1972), accompanied by numerous hydrodynamic effects.

While there is a wealth of observational evidence of onesided, clearly ram-pressure-stripped gas tails in (1) $\mathrm{H} \mathrm{I}$ in the Virgo cluster (e.g., Kenney et al. 2004; Chung et al. 2007, 2009; Abramson et al. 2011; Kenney et al. 2014) and more distant clusters (A1367; Scott et al. 2010, 2012); (2) diffuse $\mathrm{H} \alpha$ tails (Gavazzi et al. 2001; Cortese et al. 2006, 2007; Sun et al. 2007; Yagi et al. 2007; Yoshida et al. 2004, 2008; Kenney et al. 2008; Fossati et al. 2012); (3) young stars seen either in $\mathrm{H} \alpha$ or UV

\footnotetext{
* Based on observations made with ESO telescopes at La Silla Paranal Observatory under program ID 088.B-0934.
}

(Cortese et al. 2006; Sun et al. 2007; Yoshida et al. 2008; Smith et al. 2010; Hester et al. 2010; Yagi et al. 2013; Ebeling et al. 2014); and (4) X-rays (Wang et al. 2004; Finoguenov et al. 2004; Machacek et al. 2005; Sun \& Vikhlinin 2005; Sun et al. 2006, 2010), no example of a prominent (cold) molecular onesided tail is known up to now, except a few cases of extraplanar molecular gas located fairly close to the disk (NGC 4438, NGC 4522; Vollmer et al. 2005, 2008). Recently, Jáchym et al. (2013) searched for cold molecular gas in the star-forming, ram-pressure-stripped tail of the Virgo dwarf galaxy IC3418, which is (almost) completely stripped, but reached only sensitive upper limits. The presence of molecular gas has been known in some intracluster regions not connected to ram-pressurestripped tails though, as revealed by Carbon monoxide (CO) emission, which is most widely used tracer of the molecular interstellar medium. Observations of filaments near cluster cores have revealed anomalously strong $\mathrm{H}_{2}$ emission that may extend to large distances from the cD galaxies (Johnstone et al. 2007; Salomé et al. 2011). Also, in the off-disk locations of the Virgo cluster galaxy M86, Dasyra et al. (2012) found CO emission in the long $\mathrm{H} \alpha$ tidal trail that connects M86 with NGC 4438.

One of the main issues in galaxy formation and evolution is understanding the star formation history, and in particular how star formation is triggered, and how it can be suddenly quenched. Environmental effects could play an important role in this matter and, in particular, ram pressure stripping of the gas of spiral galaxies. Stars form from cold molecular interstellar gas gathered into giant molecular clouds (GMCs; e.g., Wong \& Blitz 2002). While H I can be easily stripped from galaxies by the ICM wind, the GMCs are less efficiently 


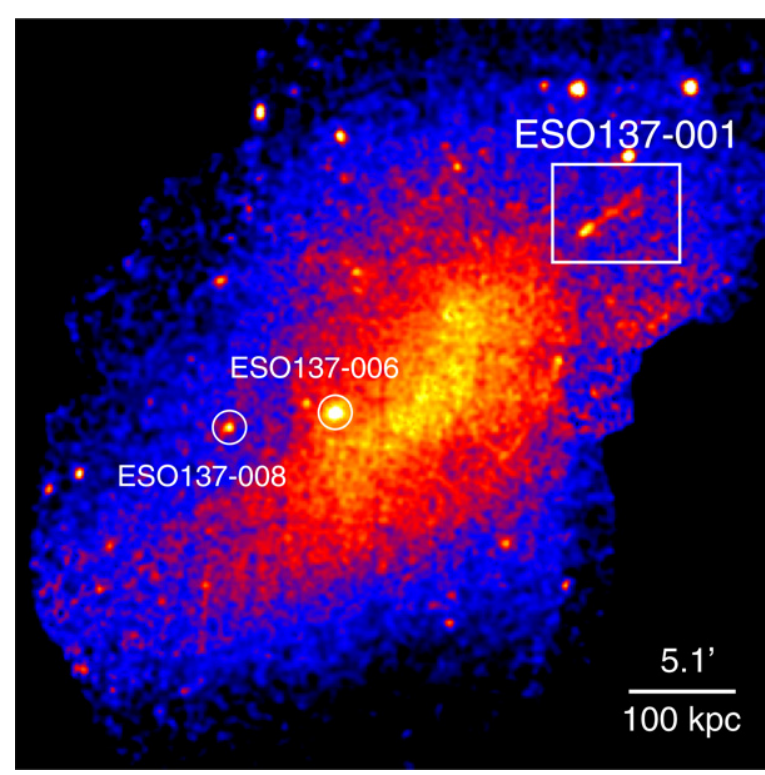

Figure 1. Central part of the A3627 cluster in a $0.5-2 \mathrm{keV}$ XMM image. The left circle marks the position of ESO 137-008, and the right circle marks the position of ESO 137-006, two galaxies in the central region of the cluster with the same 2MASS $K_{\mathrm{s}}$-band magnitude. The position of ESO 137-001 is marked with a rectangle. We zoom in into this region in Figure 2.

(A color version of this figure is available in the online journal.)

removed since they are typically denser and distributed with a higher concentration to the galactic center. Very recently, Boselli et al. (2014) found evidence that Virgo cluster galaxies that are Hi-deficient also have, on average, somewhat less molecular gas than $\mathrm{H}$ I-normal field galaxies. The effective $\mathrm{H}_{2}$ ram pressure stripping in these galaxies may be a consequence of the removal of diffuse $\mathrm{H}$ i that normally feeds giant molecular clouds. Moreover, hydrodynamic ablation by the ICM wind is assumed to assist the effective $\mathrm{H}_{2}$ stripping (Nulsen 1982; Quilis et al. 2000; Kapferer et al. 2009; Tonnesen \& Bryan 2009).

Numerical simulations predict that in the gas-stripped tails, depending on the ICM ambient pressure, radiative cooling can form in situ new dense (molecular) clouds which might then form a population of new stars (Kapferer et al. 2009; Tonnesen $\&$ Bryan 2009). If their native gas clumps were accelerated by ram pressure to velocities exceeding the escape speed from the galaxy, such stars can contribute to the intracluster light (ICL) population. In extreme environments of massive or merging galaxy clusters, it is possible that strong ram pressures could directly strip the dense molecular clouds.

\subsection{The Galaxy ESO 137-001}

The Norma cluster (A3627, $R_{\mathrm{A}}=2 \mathrm{Mpc}, M_{\mathrm{dyn}} \sim 1 \times$ $\left.10^{15} M_{\odot}, \sigma=925 \mathrm{~km} \mathrm{~s}^{-1}\right)$ is the closest $(z=0.0163)$ rich cluster comparable in mass and number of galaxies to Coma or Perseus. ${ }^{6}$ It occurs close to the center of the Great Attractor, at the crossing of a web of filaments of galaxies (called the Norma wall; Woudt et al. 2008). The Norma cluster is strongly elongated along the Norma wall, indicating an ongoing merger at the cluster core (see the central part of the cluster in Figure 1). The spiral and irregular galaxy population appears far from relaxed (Woudt et al. 2008). The center of the cluster is assumed to be at the position of the cD galaxy ESO 137-006.

\footnotetext{
6 The mean radial velocity of $4871 \pm 54 \mathrm{~km} \mathrm{~s}^{-1}$ was measured for the A3627
} cluster by Woudt et al. (2008) from radial velocities of 296 member galaxies.
Table 1

Parameters of the ESO 137-001 Galaxy

ESO 137-001 (WKK6176)

\begin{tabular}{lc}
\hline R.A., Decl. (J2000) & $16^{\mathrm{h}} 13^{\mathrm{m}} 27^{\mathrm{s}} .24,-60^{\circ} 45^{\prime} 50^{\prime \prime} 6$ \\
Type & $\mathrm{SBc}$ \\
Radial velocity & $4661 \pm 46 \mathrm{~km} \mathrm{~s}^{-1}$ \\
Major/minor diameter $^{\prime}$ & $1.26 / 0.56$ \\
P.A., inclination & $8.9,66.2$ \\
Total $^{\mathrm{a}}$ and $I$ magnitudes $^{\mathrm{b}}$ & $14.31 \pm 0.08,13.20 \pm 0.07$ \\
Stellar mass $^{\mathrm{c}}$ & $(0.5-1.4) \times 10^{10} M_{\odot}$ \\
Rotation velocity $^{\mathrm{d}}$ & $\sim 110 \mathrm{~km} \mathrm{~s}^{-1}$ \\
Luminosity distance $^{\mathrm{c}}$ & $69.6 \mathrm{Mpc}$ \\
\hline
\end{tabular}

Notes. From the HyperLeda catalog (Paturel et al. 2003).

${ }^{\text {a }}$ Sun et al. (2007) give $i=61^{\circ}-64^{\circ}$

${ }^{\mathrm{b}}$ From Sun et al. (2007); the correction for the intrinsic extinction is not applied.

${ }^{c}$ From Sun et al. (2010) and Sivanandam et al. (2010).

${ }^{\mathrm{d}}$ From the $K$-band velocity-luminosity relation (Sivanandam et al. 2010).

At a projected distance of $\sim 280 \mathrm{kpc}$ from the cluster center to the NW direction, a blue galaxy ESO 137-001 is located (see Figure 1 and Table 1). Its line-of-sight (LOS) velocity is about $-200 \mathrm{~km} \mathrm{~s}^{-1}$ with respect to the cluster mean, which suggests that the main velocity component is in the plane of the sky. Chandra and XMM-Newton imaging revealed a long, narrow $\mathrm{X}$-ray tail extending from the galaxy to $\sim 80 \mathrm{kpc}$ projected distance (Sun et al. 2006); see the left panel of Figure 2. It points away from the direction to the cluster center. The total estimated X-ray gas mass is about $10^{9} M_{\odot}$. Deeper Chandra observations revealed even a fainter secondary X-ray tail that is well separated from the primary one (Sun et al. 2010). The bright X-ray emission in the tails is presumably arising from the mixing of cold stripped ISM with the surrounding hot ICM (Sun et al. 2010; Tonnesen et al. 2011). Currently, Ruszkowski et al. (2014) suggested that the tail bifurcation may arise as a consequence of magnetized ram pressure wind which produces a very filamentary morphology of the tail.

Optical images of ESO 137-001 reveal a $\sim 40 \mathrm{kpc}$ long $\mathrm{H} \alpha$ tail comprised of diffuse gas and discrete $\mathrm{H}$ II regions roughly coincident with the X-ray tail. Moreover, a number of discrete $\mathrm{H}$ II regions are occurring outside the visible X-ray tail, forming a broader tail (Sun et al. 2007). A large amount of star formation corresponding to the total number of more than $30 \mathrm{H}$ iI regions is thus occurring well outside the main body of the galaxy. The total mass of starbursts in the $\mathrm{H}$ II regions in the tail is estimated to about $10^{7} M_{\odot}$. ESO $137-001$ is expected to initially host a significant amount of $\mathrm{HI}$ and $\mathrm{H}_{2}$. To a weak limit of $5 \times 10^{8} M_{\odot}$, no H I was detected with the Australia Telescope Compact Array (ATCA; Vollmer et al. 2001). ${ }^{7}$ Spitzer Infrared Spectrometer (IRS) observations, however, revealed more than $10^{7} M_{\odot}$ of warm $\mathrm{H}_{2}(\sim 160 \mathrm{~K})$ in the galaxy and inner $20 \mathrm{kpc}$ of the tail (Sivanandam et al. 2010). As this warm gas is likely a small fraction of the total molecular hydrogen, deep H I and $\mathrm{CO}$ observations are expected to reveal the cold component in the galaxy.

ESO 137-001 is an excellent candidate for an ongoing transformation from a blue, gas-rich to a red, gas-poor galaxy due to the violent removal of its ISM by ram pressure stripping. It has the most dramatic tail of a late-type galaxy observed up

\footnotetext{
7 The upper limit is lower than in Vollmer et al. (2001), since a lower luminosity distance of $69.6 \mathrm{Mpc}$ is adopted for the A3627 cluster. It is possible that some (extended) H I emission was filtered out by the ATCA interferometric observation. No single-dish data are available.
} 

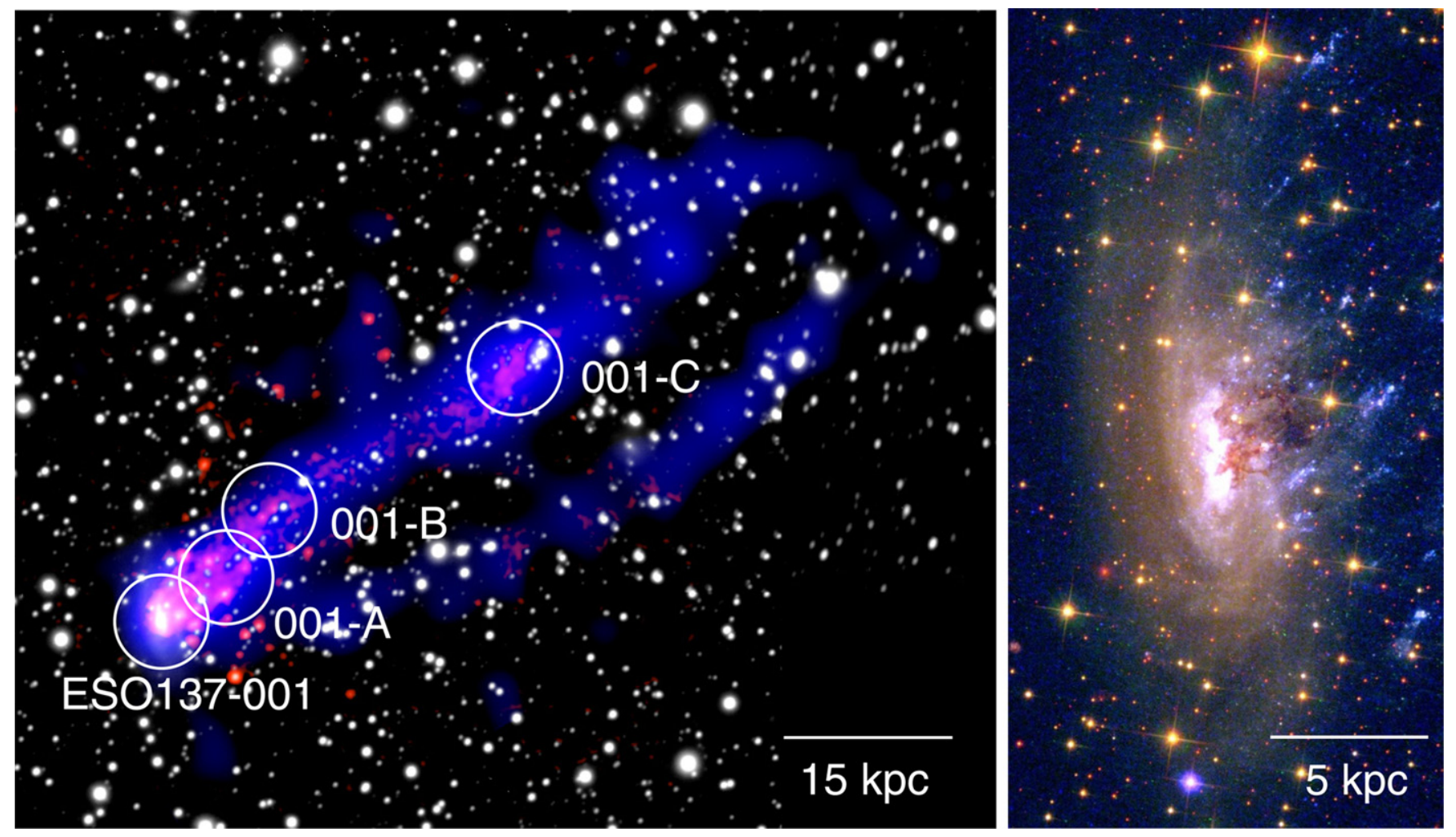

Figure 2. Left: observed positions on a composite X-ray Chandra (blue) / H $\alpha$ SOAR (red) image of ESO 137-001 with ${ }^{12} \mathrm{CO}(2-1)$ beams $\left(\mathrm{FWHM}=25^{\prime \prime} \approx 8.4 \mathrm{kpc}\right)$ of the APEX telescope. Nearly all of the optical sources are foreground stars. Right: HST image of the disk of ESO 137-001 showing the extent of the stellar component and with great detail the filamentary structure of extraplanar dust and young stars. The 3D orientation of the galaxy with respect to the observer is likely the following: the observed spiral arms are trailing; the near side of the disk is to the east (as suggested from dust extinction in the west); thus, the southern side is receding (positive radial velocities) while the northern is approaching.

(A color version of this figure is available in the online journal.)

to today, making it an ideal laboratory for detailed studies of the complex processes that take place during ram pressure stripping. A3627 is a rich cluster where ram pressure can be 1-2 orders of magnitude stronger than in Virgo. The high-pressure (including thermal pressure) environment might therefore be strong enough to affect even denser (molecular) gas. Hubble Space Telescope (HST) observations revealed a complex structure with disrupted dust content, filaments of young stars, and bright extraplanar star clusters in the inner tail of ESO 137-001 (see Figure 2, right panel). A detailed study of these observations will be published elsewhere. Herschel PACS and SPIRE imaging shows a dust trail emanating from the galaxy that is coaligned with the warm $\mathrm{H}_{2}, \mathrm{H} \alpha$, and the more prominent of the two X-ray tails (S. Sivanandam et al., in preparation).

In this paper, we aim to answer basic questions about the presence, amount, spatial distribution, and origin of molecular gas in the star-forming stripped tail of ESO 137-001. With the Actama Pathfinder EXperiment (APEX) telescope, we searched for ${ }^{12} \mathrm{CO}(2-1)$ emission in regions covering the main body of the galaxy, as well as in the $\mathrm{H} \alpha$ emission peaks in the tail. The paper is structured as follows. Observations performed are introduced in Section 2, the results are presented in Section 3, the efficiency of star formation in the system is studied in Section 4, the structure of the stripped gas is analyzed in Section 5, and the ram pressure operating on the galaxy is semi-analytically estimated in Section 6. A discussion follows in Section 7, and conclusions are drawn in Section 8. We adopt a cluster redshift of 0.0163 for A3627 (Woudt et al. 2008). Assuming $H_{0}=71 \mathrm{~km} \mathrm{~s}^{-1} \mathrm{Mpc}^{-1}, \Omega_{M}=0.27$, and $\Omega_{\Lambda}=0.73$, the luminosity distance is $69.6 \mathrm{Mpc}$, and $1^{\prime \prime}=0.327 \mathrm{kpc}$.

\section{OBSERVATIONS}

The observations of ESO 137-001 were carried out with the APEX 12 m antenna in 2011 September. ${ }^{8}$ Using the APEX-1 receiver of the Swedish Heterodyne Facility Instrument (SHFI), the observations were performed at the frequency of the ${ }^{12} \mathrm{CO}(2-1)\left(v_{\text {rest }}=230.538 \mathrm{GHz}\right)$ line. The eXtended Fast Fourier Transform Spectrometer (XFFTS) backend was used with a total bandwidth of $2.5 \mathrm{GHz}$ divided into 32,768 channels. The corresponding velocity resolution is about $0.1 \mathrm{~km} \mathrm{~s}^{-1}$. The XFFTS consists of two units with a $1 \mathrm{GHz}$ overlap region. It thus covers the entire IF bandwidth of the SHFI. At $230 \mathrm{GHz}$, the FWHM of the primary beam of the telescope is about $25^{\prime \prime}$, which, with the adopted distance of the Norma Cluster of $\sim 69.6 \mathrm{Mpc}$, corresponds to about $8.4 \mathrm{kpc}$. The main beam-projected area is $\Omega_{B} \simeq 700 \operatorname{arcsec}^{2}=80 \mathrm{kpc}^{2}$, including a factor $1 / \ln 2$ of a Gaussian beamshape correction. The system temperatures were typically about $140 \mathrm{~K}$ and the data are of excellent quality.

The observations were performed in a symmetric Wobbler switching mode with a maximum throw of $300^{\prime \prime}$ in order to avoid with OFF positions the tail if oriented in azimuth. The Wobbler switching is known to provide better baseline stability than position switching. The integration points in ESO 137001 were selected to cover its main body, as well as $\mathrm{H} \alpha$-bright regions in its tail, including one at a large projected distance of $\sim 2^{\prime}$ from the galaxy (see the scheme in Figure 2). The list of

\footnotetext{
8 In the same APEX program, the Norma ram-pressure-stripped galaxy ESO 137-002 (Sun et al. 2010) was also observed. The results will be discussed in a forthcoming paper.
} 

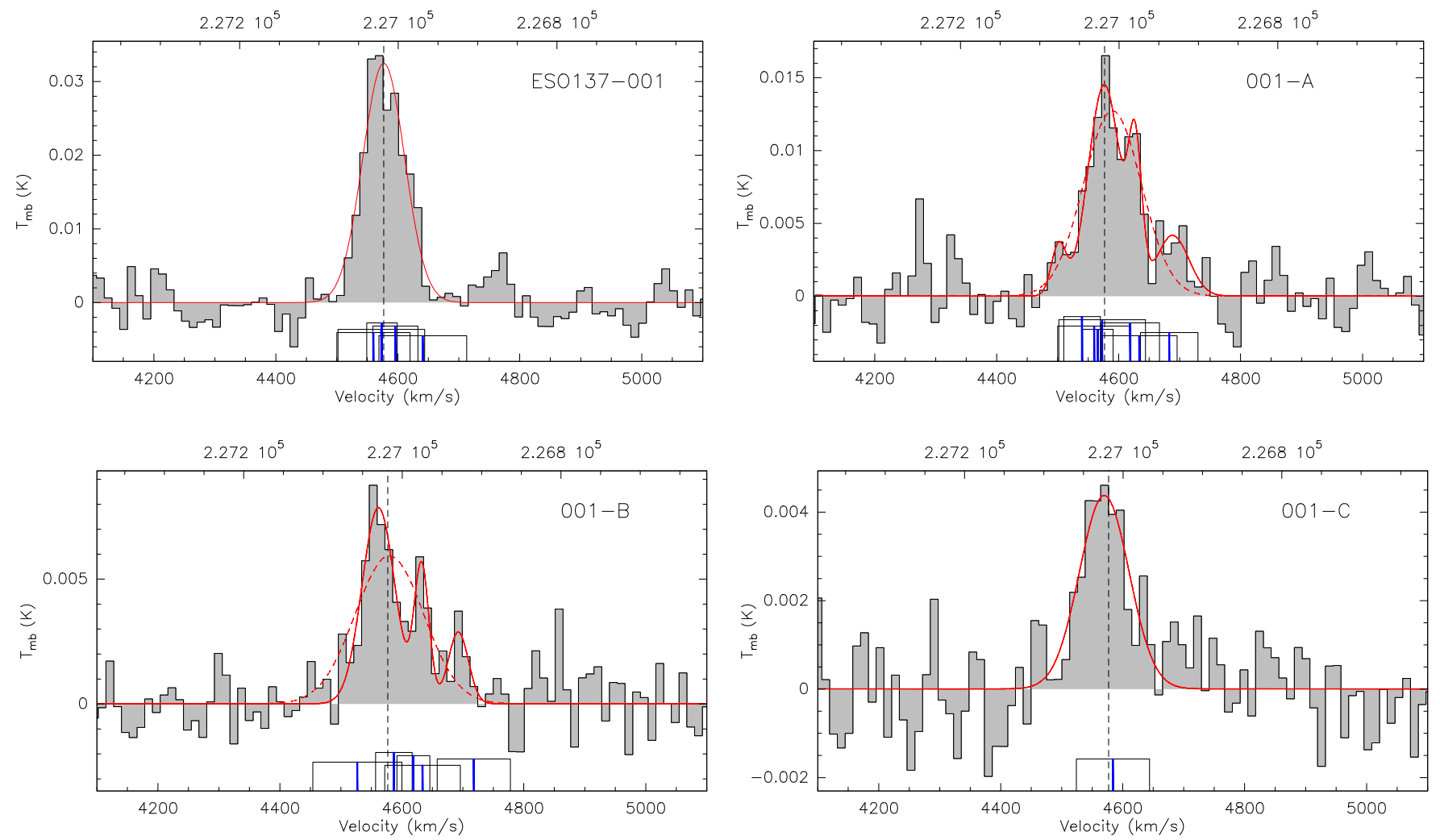

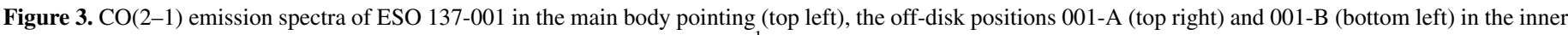

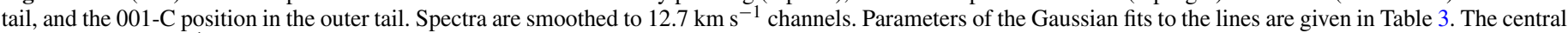

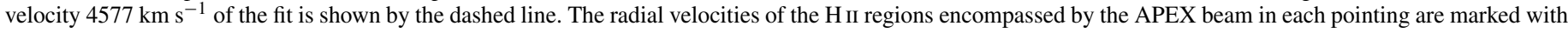
blue ticks with wings corresponding to the velocity errors (Sun et al. 2007). Their heights order the regions according to H $\alpha$ luminosity.

(A color version of this figure is available in the online journal.)

Table 2

List of Observed Positions

\begin{tabular}{lcccc}
\hline \hline Source & $\begin{array}{c}\text { R.A. } \\
(\mathrm{J} 2000)\end{array}$ & $\begin{array}{c}\text { Decl. } \\
(\mathrm{J} 2000)\end{array}$ & $\begin{array}{c}v_{\text {lsr, opt }} \\
\left(\mathrm{km} \mathrm{s}^{-1}\right)\end{array}$ & $\begin{array}{r}T_{\mathrm{ON}} \\
(\mathrm{min})\end{array}$ \\
\hline ESO137-001 & $16: 13: 27.24$ & $-60: 45: 50.6$ & 4630 & 13 \\
001-A & $16: 13: 24.75$ & $-60: 45: 39.5$ & 4600 & 21 \\
001-B & $16: 13: 23.30$ & $-60: 45: 21.0$ & 4600 & 56 \\
001-C & $16: 13: 14.34$ & $-60: 44: 42.4$ & 4500 & 137 \\
\hline
\end{tabular}

observed positions is given in Tab. 2, together with information on their radial velocity and actual on-source observing time. The receiver was tuned to the ${ }^{12} \mathrm{CO}(2-1)$ frequency shifted in each position to its respective optical radial velocity (given in Table 2). Observing conditions were good with PWV less than $1 \mathrm{~mm}$.

The data were reduced according to the standard procedure using CLASS from the GILDAS software package developed at IRAM. Bad scans were flagged and emission-line-free channels in the total width of about $1000 \mathrm{~km} \mathrm{~s}^{-1}$ were used to subtract first-order baselines. The corrected antenna temperatures, $T_{\mathrm{A}}^{*}$, provided by the APEX calibration pipeline (Dumke \& Mac-Auliffe 2010) were converted to main-beam brightness temperatures by $T_{\mathrm{mb}}=T_{\mathrm{A}}^{*} / \eta_{\mathrm{mb}}$ using a main beam efficiency of about $\eta_{\mathrm{mb}}=0.75$ at $230 \mathrm{GHz}$. rms noise levels typically of 1-2 mK per $12.7 \mathrm{~km} \mathrm{~s}^{-1}$ channels were obtained. Gaussian fits were used to measure the peak $T_{\mathrm{mb}}$, width, and position of the detected $\mathrm{CO}$ lines. Flux densities can be obtained using the conversion factor $S_{v} / T_{m b}=39 \mathrm{Jy}$ beam $^{-1} \mathrm{~K}^{-1}$ for the APEX telescope.

\section{RESULTS}

\subsection{Main Body of ESO 137-001}

In the pointing centered on the main body of ESO 137-001, $\mathrm{CO}(2-1)$ emission is strongly detected (see Figure 3, top left panel). The corresponding molecular gas mass is about $1.1 \times$ $10^{9} M_{\odot}$, following $M_{\mathrm{H}_{2}}\left[M_{\odot}\right]=5.5 L_{\mathrm{CO}(2-1)}\left[\mathrm{K} \mathrm{km} \mathrm{s}^{-1} \mathrm{pc}^{2}\right]$, where we assume typical values for the $\mathrm{CO}(2-1)$-to- $\mathrm{CO}(1-0)$ ratio of 0.8 (e.g., Leroy et al. 2009), and a standard Galactic $\mathrm{CO}(1-0)$-to- $\mathrm{H}_{2}$ conversion factor of $2 \times 10^{20} \mathrm{~cm}^{-2}\left(\mathrm{~K} \mathrm{~km} \mathrm{~s}^{-1}\right)^{-1}$ (e.g., Kennicutt \& Evans 2012; Feldmann et al. 2012). The formula includes a factor of 1.36 to account for the presence of Helium. Using a Gaussian fit (plotted in Figure 3 over the spectrum), we measure the linewidth of $81 \mathrm{~km} \mathrm{~s}^{-1}$ and the central velocity of the line of $\sim 4577 \mathrm{~km} \mathrm{~s}^{-1}$, assuming the radio definition of the velocity. ${ }^{9}$ Other parameters of the line are given in Table 3. Sun et al. (2007) measured the radial velocity of the central emission nebula from two different optical CTIO $1.5 \mathrm{~m}$ spectra to $4667 \pm 135 \mathrm{~km} \mathrm{~s}^{-1}$ and $4640 \pm 20 \mathrm{~km} \mathrm{~s}^{-1}$, which is very close to our measurement. ${ }^{10}$

As is apparent from Figure 2, the central APEX beam has been contaminated by a contribution from off-disk regions. Within its $\sim 8 \mathrm{kpc}$ FWHM, it encompasses not only the central $\mathrm{H} \alpha$ emission nebula but also five identified off-disk $\mathrm{H}$ II regions (Sun et al. 2007), including the brightest one (ELO1 in Sun et al. 2007) that is located only about $2 \mathrm{kpc}$ downstream in the tail. Their radial velocities are mostly close $\left(\lesssim 30 \mathrm{~km} \mathrm{~s}^{-1}\right)$

\footnotetext{
$9 v_{\text {rad }}=c\left(1-v_{\text {sky }} / v_{0}\right) \approx 4577 \mathrm{~km} \mathrm{~s}^{-1}$

$10 v_{\mathrm{opt}}=c\left(v_{0} / v_{\mathrm{sky}}-1\right) \approx 4648 \mathrm{~km} \mathrm{~s}^{-1}$.
} 
Table 3

Properties of the Detected $\mathrm{CO}(2-1)$ Lines

\begin{tabular}{|c|c|c|c|c|c|c|c|}
\hline Source & $\begin{array}{c}\mathrm{rms} \\
(\mathrm{mK})\end{array}$ & $\begin{array}{l}\text { Velocity } \\
\left(\mathrm{km} \mathrm{s}^{-1}\right)\end{array}$ & $\begin{array}{c}\text { FWHM } \\
\left(\mathrm{km} \mathrm{s}^{-1}\right)\end{array}$ & $\begin{array}{l}T_{\text {peak }} \\
(\mathrm{mK})\end{array}$ & $\begin{array}{c}I_{\mathrm{CO}, \mathrm{fit}} \\
\left(\mathrm{K} \mathrm{km} \mathrm{s}^{-1}\right)\end{array}$ & $\begin{array}{c}I_{\mathrm{CO}} \\
\left(\mathrm{K} \mathrm{km} \mathrm{s}^{-1}\right)\end{array}$ & $\begin{array}{c}M_{\mathrm{mol}} \\
\left(10^{8} M_{\odot}\right)\end{array}$ \\
\hline ESO137-001 & 2.6 & $4576.9 \pm 1.8$ & $80.7 \pm 4.0$ & 32.5 & $2.8 \pm 0.1$ & 2.7 & 11.1 \\
\hline \multirow[t]{5}{*}{ 001-A } & 1.9 & $4589.6 \pm 4.0$ & $111.8 \pm 10.9$ & 12.7 & $1.5 \pm 0.1$ & 1.6 & 6.4 \\
\hline & & $4501.4 \pm 8.7$ & $28.6 \pm 21.3$ & 3.5 & $0.11 \pm 0.07$ & - & 0.5 \\
\hline & & $4576.3 \pm 3.7$ & $62.5 \pm 12.5$ & 14.5 & $0.97 \pm 0.15$ & - & 4.0 \\
\hline & & $4626.7 \pm 3.6$ & $25.7 \pm 7.7$ & 9.3 & $0.25 \pm 0.11$ & - & 1.0 \\
\hline & & $4688.3 \pm 9.9$ & $63.2 \pm 22.2$ & 4.2 & $0.28 \pm 0.09$ & - & 1.2 \\
\hline \multirow[t]{4}{*}{ 001-B } & 1.2 & $4579.4 \pm 6.3$ & $132.5 \pm 16.8$ & 5.9 & $0.84 \pm 0.08$ & 0.8 & 3.4 \\
\hline & & $4561.3 \pm 3.4$ & $63.4 \pm 11.2$ & 7.9 & $0.53 \pm 0.07$ & - & 2.2 \\
\hline & & $4632.2 \pm 4.2$ & $28.4 \pm 14.8$ & 5.5 & $0.17 \pm 0.07$ & - & 0.7 \\
\hline & & $4692.3 \pm 8.7$ & $39.0 \pm 29.7$ & 2.9 & $0.12 \pm 0.06$ & - & 0.5 \\
\hline 001-C & 1.0 & $4569.5 \pm 5.5$ & $95.9 \pm 17.0$ & 4.4 & $0.45 \pm 0.06$ & 0.4 & $1.5-1.8$ \\
\hline
\end{tabular}

Notes. This table provides the $1 \sigma \mathrm{rms}$ in the $12.7 \mathrm{~km} \mathrm{~s}^{-1}$ channels, the parameters of (multiple) Gaussian fits (the line heliocentric central velocity, FWHM, peak temperature, and the integrated intensity), the measured integrated intensity, and the molecular gas mass. The temperatures are given in $T_{\mathrm{mb}}$ scale. First order baselines were subtracted in the velocity range $4100-5100 \mathrm{~km} \mathrm{~s}^{-1}$. The radio definition of velocity was used to convert the sky frequency of the source.

to the galaxy's system velocity, except one at $54 \pm 72 \mathrm{~km} \mathrm{~s}^{-1}$ located near the southern beam edge. The radial velocities of the encompassed H II regions are shown at the bottom of the spectrum panel in Figure 3 with the blue bars with black wings corresponding to the errors. The ratio of $\mathrm{H} \alpha$ luminosities of the central nebula and of the brightest off-disk region ELO1 is $\sim 10$ (Sun et al. 2007). This suggests that the central nebula could also dominate the $\mathrm{CO}$ emission in the main body pointing; however, we do not know the relation between $\mathrm{H} \alpha$ and $\mathrm{CO}$ emission and thus cannot say what fraction of the total $\mathrm{H}_{2}$ mass of $\sim 1.1 \times$ $10^{9} M_{\odot}$ detected in the main body point belongs to the off-disk sources.

\subsection{Molecular Gas in the Halo-The Inner Tail of ESO 137-001}

In the innermost tail pointing 001-A, located roughly one beam $(\sim 8 \mathrm{kpc})$ downstream of the galaxy center, $\mathrm{CO}(2-1)$ emission is detected with very high significance (see Figure 3, top right panel). The measured $\mathrm{CO}$ luminosity corresponds to $6.4 \times 10^{8} M_{\odot}$ of molecular gas, about half the mass found in the main body position. Although the $\mathrm{X}$-factor is uncertain for the special environment of a gas stripped tail, we assume the Galactic value because the metallicity measured in the tail is close to solar $\left(\sim 0.6 Z_{\odot}\right.$, Sun et al. 2010). The actual value of the $\mathrm{X}$-factor will be discussed in a forthcoming paper based on observations of other $\mathrm{CO}$ transitions in the system. Multiple components are visible in the 001-A line-a wing at the lowvelocity side, a double-profile main part, and a $4 \sigma$ feature at the high-velocity side centered at $\sim 4687 \mathrm{~km} \mathrm{~s}^{-1}$. In Table 3 , the parameters of the multiple Gaussian fits are given together with a single Gaussian fit of the whole line. The central velocity of the single fit is only slightly higher $\left(\sim 4590 \mathrm{~km} \mathrm{~s}^{-1}\right)$ than in the main body position. We discuss the components in more detail in Section 7.1. The 001-A pointing encompasses three bright $\mathrm{H}$ II regions at radial velocities $-50 \pm 30,-23 \pm 25$, and $31 \pm 48 \mathrm{~km} \mathrm{~s}^{-1}$ with respect to the galaxy's systemic velocity, as well as several weaker regions, including one at the edge of the beam that has a higher radial velocity $\left(97 \pm 47 \mathrm{~km} \mathrm{~s}^{-1}\right)$ which corresponds well to the high-velocity $4 \sigma$ feature in the CO spectrum.
Advancing another $\sim 8 \mathrm{kpc}$ along the tail to the $001-\mathrm{B}$ point, about $3.4 \times 10^{8} M_{\odot}$ of molecular gas is detected (see Figure 3, bottom left panel), about half of the amount found in 001-A. The line profile again shows several velocity components that are more distinct than in the case of 001-A. The parameters of a multiple Gaussian profile fit are given in Table 3. Although not included in the fitting, there is a hint of a low-velocity feature similar to that in 001-A. The 001-B pointing encompasses several bright $\mathrm{H}$ II regions that are moving at about $-64 \mathrm{~km} \mathrm{~s}^{-1}$ to $+133 \mathrm{~km} \mathrm{~s}^{-1}$ relative to the systemic velocity along the line of sight. Thus, $\mathrm{CO}$ and $\mathrm{H} \alpha$ velocities are again well consistent.

In total, in the inner tail regions 001-A and 001-B, we thus detected almost the same amount ( $\sim 90 \%)$ of molecular gas as in the galaxy pointing. Taking into account the contribution of offdisk regions to the main body point intensity, and the uncovered parts of the tail, there probably is more $\mathrm{H}_{2}$ in the inner $\sim 20 \mathrm{kpc}$ of the tail than in the main body.

\subsection{IC Molecular Gas-The Outer Tail of ESO 137-001}

Strong $\mathrm{CO}(2-1)$ emission is detected even in the outermost position we have searched located at $\sim 40 \mathrm{kpc}$ (in projection) from the main body, where both X-ray and $\mathrm{H} \alpha$ emission peak (see Figure 3, bottom right panel). This is the first time $\mathrm{CO}$ emitting gas is detected in intra-cluster regions of a clearly ram-pressure-stripped tail, which moreover is spatially associated with star-forming regions and X-ray-emitting gas. The corresponding molecular gas mass is $\sim 1.8 \times 10^{8} M_{\odot}$, which is about a factor of 2 less than in the region 001-B, and it constitutes about $15 \%$ of the total $\mathrm{H}_{2}$ mass found in the tail. As we will discuss later in Section 5.2, a higher molecular gas temperature in the tail (as well as optical thickness and other effects), especially at the farthest pointing 001-C, could possibly decrease the $\mathrm{CO}$-to- $\mathrm{H}_{2}$ conversion factor from a Galactic value that we used and therefore yield an overestimate of the $\mathrm{H}_{2}$ content (Bolatto et al. 2013).

A single Gaussian fit of the $\mathrm{CO}$ line is centered at $\sim 4570 \mathrm{~km} \mathrm{~s}^{-1}$, which is very close to the central velocity of the main body line. This indicates that the gas-stripped tail indeed extends almost entirely in the plane of the sky and also suggests that the orbital motion of the galaxy occurs mostly in 
Table 4

The H $\alpha$-based SFRs in the Observed Regions

\begin{tabular}{lccc}
\hline \hline Source & $\begin{array}{c}L_{\mathrm{H} \alpha} \\
\left(\mathrm{erg} \mathrm{s}^{-1}\right)\end{array}$ & $\begin{array}{c}\mathrm{SFR}_{\mathrm{H} \alpha} \\
\left(M_{\odot} \mathrm{yr}^{-1}\right)\end{array}$ & $\begin{array}{c}L_{\mathrm{CO}} \\
\left(\mathrm{K} \mathrm{km} \mathrm{s}^{-1} \mathrm{pc}^{2}\right)\end{array}$ \\
\hline ESO137-001 & $6.7 \times 10^{40}$ & $3.6 \times 10^{-1}$ & $2.0 \times 10^{8}$ \\
001-A & $6.3 \times 10^{39}$ & $3.4 \times 10^{-2}$ & $1.1 \times 10^{8}$ \\
001-B & $1.4 \times 10^{39}$ & $7.5 \times 10^{-3}$ & $5.0 \times 10^{7}$ \\
001-C & $1.7 \times 10^{38}$ & $9.2 \times 10^{-4}$ & $4.0 \times 10^{7}$ \\
\hline
\end{tabular}

Notes. The $\mathrm{H} \alpha$ luminosities from $\mathrm{H}$ II regions covered with our APEX pointings (circles with a diameter of $25^{\prime \prime}$ ) and corresponding star formation rates, together with measured CO luminosity.

the plane of the sky. Table 3 further summarizes the parameters of the line fit. The CO line profile is asymmetric, again indicating an internal structure. Only one $\mathrm{H}$ II region identified by Sun et al. (2007) occurs in the 001-C pointing area. Its radial velocity is well consistent with the central velocity of the $\mathrm{CO}$ line (see the blue bar in the bottom right panel of Figure 3).

The tidal truncation radius of the dark matter halo of ESO 137-001 is estimated to be about $15 \mathrm{kpc}$ (Sun et al. 2010). This suggests that the molecular gas detected in the 001-C region could already be released from the gravitational well of the galaxy and thus contribute directly to the intracluster material in the Norma cluster. Due to the lack of (almost) any radial velocity component, however, we cannot determine whether the detected molecular gas is indeed at velocities exceeding the escape speed from the galaxy or stays bound to it.

\section{EFFICIENCY OF STAR FORMATION}

The optical spectroscopy and the $\mathrm{H} \alpha$ imaging revealed starforming nucleus of ESO 137-001, as well as over $30 \mathrm{HII}$ regions downstream in the tail (Sun et al. 2007, 2010). Subsequent infrared data showed associated $8 \mu \mathrm{m}$ excess sources in the inner $\sim 1^{\prime}$ of the tail (Sivanandam et al. 2010). In this section, we estimate how efficient the transformation of the detected molecular gas into stars has been. We are especially interested in the situation in the gas-stripped tail of the galaxy.

\subsection{Star Formation in the Disk}

The rate of ongoing star formation (SFR) corresponding to the $\mathrm{H} \alpha$ luminosity of the main body of ESO 137-001 can be calculated from the Kennicutt \& Evans (2012) relation

$$
\log \operatorname{SFR}\left(M_{\odot} \mathrm{yr}^{-1}\right)=\log L_{\mathrm{H} \alpha}\left(\mathrm{erg} \mathrm{s}^{-1}\right)-41.27
$$

which uses the initial mass function (IMF) of Kroupa \& Weidner (2003). The $\mathrm{H} \alpha$ luminosity of $\mathrm{H}$ II regions covered by the ESO 137-001 main body APEX pointing is $\sim 6.7 \times 10^{40} \mathrm{erg} \mathrm{s}^{-1}$ (see Table 4), dominated by the central nebula. The corresponding SFR is $\sim 0.4 M_{\odot} \mathrm{yr}^{-1}$. It has been shown that multiwavelength observations may reduce uncertainties and errors in SFR determination caused mainly by dust attenuation, especially for edge-on galaxies. While there is no data for ESO 137-001 from the Spitzer/Multiband Imaging Photometer (MIPS), the WideField Infrared Survey Explorer (WISE) measured in its band 4 $(22 \mu \mathrm{m})$ a flux density of $58.7 \mathrm{mJy}$. Using the SFR WISE calibration relations of Lee et al. (2013) corrected for the Kroupa IMF, in combination with the $\mathrm{H} \alpha$ flux of the galaxy (Sun et al. 2007), the corresponding SFR of the main body of ESO 137-001 is $\sim 1 M_{\odot} \mathrm{yr}^{-1}$.

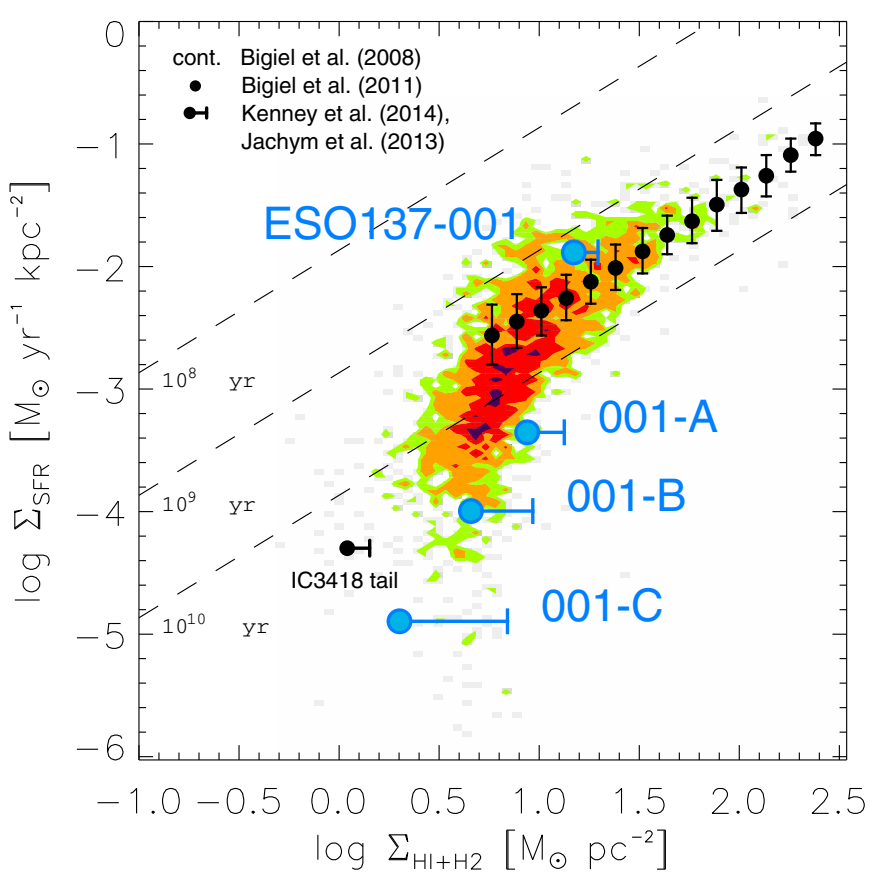

Figure 4. SFR surface density as a function of total gas surface density in ESO 137-001 and the three observed tail regions. The filled circles correspond to the molecular gas column density, while the error bars show ATCA H i upper limits. For comparison, measurements from subkiloparsec sampling of disks of nearby spiral galaxies (Bigiel et al. 2008) are shown, with $\Sigma_{\text {SFR }}$ inferred from $\mathrm{H} \alpha$ emission. The contours correspond to pixel-by-pixel sampling of Bigiel et al. (2008) of the optical disks of seven nearby spirals: contours of $1,2,5$, and 10 sampling points per cell ( 0.05 dex size) correspond to green, orange, red, and magenta, respectively. The black dots show the average molecular gas depletion time measured in 30 nearby galaxies (IRAM HERACLES CO survey, Bigiel et al. 2011). For comparison, the situation in the outer parts of the star-forming tail of the Virgo IC3418 dwarf galaxy where $\mathrm{H}$ I was detected and $\mathrm{H}_{2}$ measured to upper limits (Kenney et al. 2014; Jáchym et al. 2013) is also shown. Figure adapted from Bigiel et al. (2008).

(A color version of this figure is available in the online journal.)

In Figure 4, the deduced SFR in ESO 137-001 averaged over the observed APEX $230 \mathrm{GHz}$ beam area $\left(\sim 80 \mathrm{kpc}^{2}\right)$ is plotted into a Kennicutt-Schmidt-type plot showing $\Sigma_{\mathrm{SFR}}$ as a function of the total (molecular + atomic) gas column density. In the plot, measurements from disks of seven nearby spiral galaxies sampled at sub-kiloparsec scales by Bigiel et al. (2008, their Figure 8) are shown for comparison. The filled circle at ESO 137-001's position in Figure 4 corresponds to the $\mathrm{H}_{2}$ column density, while the ATCA H I upper limit is displayed with a horizontal error bar. Thus, in the main body of ESO 137-001, the measured molecular gas depletion time (i.e., consumption timescale due to star formation, also known as the inverse of the SFE) is well consistent with average values found in other nearby spiral galaxies $\left(\tau_{\mathrm{dep}, \mathrm{H}_{2}} \approx 2.35 \mathrm{Gyr}\right.$, Bigiel et al. 2011, see black dots in Figure 4). The (total) gas surface density of $\sim 9 M_{\odot} \mathrm{pc}^{-2}$ was identified as a limit below which the SFE starts to vary substantially (at a given gas surface density). Bigiel et al. (2008) propose that this is due to local conditions, such as metallicity, gas pressure, etc., that begin to play a role. In such places, $\Sigma_{\mathrm{HI}}>\Sigma_{\mathrm{H}_{2}}$ typically. The averaged $\mathrm{H}_{2}$ column density in the main body ESO 137-001 pointing exceeds this limit.

\subsection{Star Formation in the Tail}

In the gas-stripped tail of ESO 137-001, the only observed $\mathrm{SF}$ tracer in all our APEX regions is $\mathrm{H} \alpha$ emission that gives 
evidence of recent star formation over the last $\sim 10 \mathrm{Myr}^{11}$ Table 4 summarizes $\mathrm{H} \alpha$ luminosities of $\mathrm{H}$ II regions covered by our APEX pointings (circles with a $25^{\prime \prime}$ diameter). In Table 4 , a 1 mag extinction correction was applied, which is probably too high for the tail regions, however, this only makes the conclusions of this section stronger. Following Equation (1), the SFR in the innermost tail region 001-A is low, $\sim 0.03 M_{\odot} \mathrm{yr}^{-1}$. It further drops by a factor of 4.5 when going to region $001-\mathrm{B}$, and by another factor of $\sim 8$ in the most distant IC position 001-C, down to $\sim 10^{-3} M_{\odot} \mathrm{yr}^{-1}$ (see Table 4). The total SFR in the three tail regions is $\sim 0.04 M_{\odot} \mathrm{yr}^{-1}$. This amount is consistent with predictions of numerical simulations by Tonnesen \& Bryan (2012), who found the total SFR to be saturated at $\sim 0.05 M_{\odot} \mathrm{yr}^{-1}$ in the ram-pressure-stripped tail of their (massive) model galaxy. The low levels of star formation in the inner tail are also suggested from Spitzer/MIPS $24 \mu \mathrm{m}$ band observations of Sivanandam et al. (2010), who measured in a region that covers partially our 001-A and 001-B apertures a flux of less than $10 \%$ of the value from their nuclear region that roughly corresponds to our ESO 137-001 aperture.

The $\mathrm{H} \alpha$-based star formation rates averaged over the APEX beams are for the observed tail pointings also placed into the Kennicutt-Schmidt-type plot in Figure 4. Again, the filled circles correspond to $\mathrm{H}_{2}$ column densities, while the ATCA upper limits on the $\mathrm{H}_{\mathrm{I}}$ mass per pointing $\left(\sim 4.7 \mathrm{M}_{\odot} \mathrm{pc}^{-2}\right)$ are taken into account and displayed with the horizontal error bars. Clearly, the location of the points 001-A, 001-B, and 001-C in Figure 4 indicate much lower efficiency of star formation in the gas-stripped tail of ESO 137-001 than is typical in starforming ISM in the inner parts of spiral disks. The molecular gas depletion times are above $10^{10} \mathrm{yr}$, thus exceeding a Hubble time, which indicates that most of the stripped gas does not form stars but remains gaseous and ultimately joins the ICM. Similarly low SFEs are found in the outer disks of spiral galaxies, in H I-rich dwarf irregular galaxies (e.g., Huang et al. 2012; Lee et al. 2009), or in low surface brightness disks (e.g., Wyder et al. 2009), where, however, H I is likely to represent most of the ISM and CO is mostly undetected (Bigiel et al. 2010). The CO-bright gas-stripped tail of ESO 137-001 (with only upper limits on the $\mathrm{H}$ I content) thus represents a special environment of SF distinct from the above typical categories.

While the Kennicutt-Schmidt law finds a tight correlation between the surface densities $\Sigma_{\mathrm{SFR}}$ and $\Sigma_{\text {gas }}$ in galactic disks for which the typical unobserved scale height is $\sim 100 \mathrm{pc}$, in the tail of ESO 137-001 the unobserved thickness is likely a few kiloparsecs. A low average density of the gas that is distributed in a wide three-dimensional (3D) tail is thus likely an important factor in the observed low SFE. Another factor could be ram-pressure-induced shocks that may increase the thermal and turbulent pressure of the stripped ISM (Sivanandam et al. 2010). Turbulence can suppress star formation by inhibiting collapse of molecular clouds.

In Figure 4, we also plot for comparison the total gas column density ( $\mathrm{H}_{\mathrm{I}}$ detected, $\mathrm{H}_{2}$ measured to upper limits) averaged over the outer parts $\left(\sim 35 \mathrm{kpc}^{2}\right)$ of the star-forming tail of the Virgo cluster ram-pressure-stripped dwarf galaxy IC3418, where the total $\mathrm{H} \alpha$ luminosity of the $\mathrm{H}$ II regions corresponds

\footnotetext{
11 We note that the classical SFR-luminosity relation is expected to work well for (1) continuous star formation approximation where SFR is constant over the timescale of $\mathrm{H} \alpha$ emission and (2) regions where $\mathrm{H} \alpha$ emission averages over more emitting sources. We are not sure how well the two conditions are fulfilled in the ESO 137-001 tail. The estimate of SFRs in the complex environments of a gas-stripped tail is thus far from trivial.
}

to a SFR of $\sim 1.9 \times 10^{-3} M_{\odot} \mathrm{yr}^{-1}$ (Kenney et al. 2014; Jáchym et al. 2013). The resulting star formation efficiency (SFE) is again lower than $10^{10} \mathrm{yr}$.

Similarly, in the ram-pressure-stripped extraplanar gas of several Virgo cluster spirals, Vollmer et al. (2012) and Boissier et al. (2012) found a depressed SFE with respect to the available amount of total gas. In one case (NGC 4438), Vollmer et al. (2012) determined a lower SFE even with respect to the molecular gas. They suggested that the stripped gas loses the gravitational confinement and associated pressure of the galactic disk, which leads to a decrease in gas density and consequently in star formation. As we will discuss later in Section 7.2, the thermal ICM pressure at the location of ESO 137-001 in the Norma cluster, however, is estimated to be similar to midplane pressures in galactic disks. Low SFEs are also known from other $\mathrm{H}_{2}$ luminous sources, such as radio galaxies where radioloud active galactic nuclei are assumed to enhance heating and turbulence in the molecular gas in disks, or, for example, in Stephan's Quintet where turbulence invoked by galaxy-tidal arm collision is a probable source of heating (e.g., Nesvadba et al. 2010; Guillard et al. 2012).

\subsection{Ram Pressure Dwarf Galaxy in the Making?}

With the detected large reservoir of $>10^{8} M_{\odot}$ of molecular gas, the (intra-cluster) region 001-C resembles tidal dwarf galaxies (TDGs; e.g., Braine et al. 2001) in that it is young, it also has been formed by condensation of pre-enriched matter that belonged to a parent galaxy, it is now (probably) decoupled, and it may be gravitationally bound. We thus speculate that it could be an example of a ram pressure dwarf galaxy (RPDG) in the making from the local collapse of abundant (stripped) gas. While in TDGs a typical molecular gas fraction is $\sim 20 \%$, in $001-\mathrm{C} \mathrm{H}_{2}$ is likely the dominant gas phase.

However, in the extreme environment of the outer gasstripped tail embedded in a hot ICM, the actual amount of molecular gas in the 001-C region may be overestimated by a galactic X-factor. Moreover, while in TDGs the efficiency of star formation was measured to be similar to spirals, it is much lower in the 001-C pointing. Also, the suggested value of SFRs in the 001-C region (of the order of $10^{-3} M_{\odot} \mathrm{yr}^{-1}$ ) is similar to values found in compact knots of collisional debris of interacting galaxies rather than in TDGs (Duc \& Renaud 2013). However, it could be that in 001-C we are only observing the onset of star formation. Thus, to decide whether within the 001-C pointing we are observing only a compact star-forming knot, or (for the first time) a ram-pressure-stripped dwarf galaxy building up, more detailed observations, including sensitive H I search, are needed to determine the total mass of the object, its kinematics, and especially its self-gravitation.

\section{GAS PHASES IN THE STRIPPED TAILS}

In order to better understand the structure of the gas-stripped tail of ESO 137-001, in this section we will summarize and compare the fractions of different gas phases in the tail as a whole, as well as search for trends along the tail by studying local variations in the observed regions.

\subsection{The Whole Tail of ESO 137-001}

In addition to our APEX detection of more than $1 \times 10^{9} M_{\odot}$ of cold molecular gas in the three observed pointings in the tail of ESO 137-001, current amounts of other gas phases in 
Table 5

Masses of Individual ISM Components in ESO 137-001

\begin{tabular}{lccccc}
\hline \hline Source & $\begin{array}{c}\text { Cold } \mathrm{H}_{2} \\
\left(10^{8} M_{\odot}\right)\end{array}$ & $\begin{array}{c}\text { Warm } \mathrm{H}_{2} \\
\left(10^{8} M_{\odot}\right)\end{array}$ & $\begin{array}{c}\mathrm{H} \mathrm{I} \\
\left(10^{8} M_{\odot}\right)\end{array}$ & $\begin{array}{c}\mathrm{H} \alpha \\
\left(10^{8} M_{\odot}\right)\end{array}$ & $\begin{array}{c}\text { X-Rays } \\
\left(10^{8} M_{\odot}\right)\end{array}$ \\
\hline body & $<11^{\mathrm{a}}$ & - & $<5^{\mathrm{b}}$ & $-^{\mathrm{c}}$ & 0.8 \\
tail & $>11^{\mathrm{a}}$ & $>0.4$ & $<24$ & 1.9 & 11 \\
001-A & 6.4 & $\sim 0.2$ & $<3.5$ & 0.8 & 1.3 \\
001-B & 3.4 & $\sim 0.1$ & $<3.5$ & 0.5 & 0.9 \\
001-C & 1.8 & - & $<3.5$ & 0.7 & 1.4 \\
\hline
\end{tabular}

Notes. Masses of individual ISM components in ESO 137-001 and its stripped tail. Volume filling factors of 0.05 and 1 are assumed for the $\mathrm{H} \alpha$ and X-ray emitting gas, respectively (see the text).

${ }^{a}$ It is likely that some fraction of $\mathrm{CO}$ emission in the main body pointing is coming from the encompassed off-disk inner tail $\mathrm{H}$ II regions.

b The ATCA upper limit of $5 \times 10^{8} M_{\odot}$ per $30^{\prime \prime}$ beam would correspond to $\mathrm{H}_{\mathrm{I}}$ mass upper limit in the optical area of the disk of $\sim 1 \times 10^{9} M_{\odot}$. However, we stick to the lower value as $\mathrm{H}$ I from outer disk radii has likely been stripped.

${ }^{c}$ The $\mathrm{H} \alpha$ disk is much brighter than the diffuse $\mathrm{H} \alpha$ emission and is considered to be mainly mixture of many $\mathrm{H}$ II regions.

the tail have been known at least to upper limits from previous observations.

1. About $1 \times 10^{9} \mathrm{f}_{\mathrm{X}}^{1 / 2} M_{\odot}$ of soft $(0.5-2 \mathrm{keV}) \mathrm{X}$-ray-emitting gas was revealed by Chandra and XMM-Newton imaging in the double-structure tail (Sun et al. 2006, 2010), where $\mathrm{f}_{\mathrm{X}}$ is the volume-filling factor of the X-ray-emitting gas that is expected to be close to 1 (Sun et al. 2010).

2. No H I was detected to a limit of $5 \times 10^{8} M_{\odot}$ per $30^{\prime \prime}$ beam with ATCA (Vollmer et al. 2001), which translates to an upper limit of $\sim 2 \times 10^{9} M_{\odot}$ for the whole tail, assuming it covers about four times the ATCA beam size.

3. Up to $5 \times 10^{8} \mathrm{f}_{\mathrm{H} \alpha}^{1 / 2} M_{\odot}$ of ionized, $\mathrm{H} \alpha$-emitting diffuse gas was found in the tail (Sun et al. 2007), assuming the $\mathrm{H} \alpha$ tail is approximated by a cylinder $40 \mathrm{kpc}$ long with a diameter of $3.5 \mathrm{kpc}$, and where $\mathrm{f}_{\mathrm{H} \alpha}$ is the volume-filling factor of the $\mathrm{H} \alpha$ gas that is expected to be low, as suggested from numerical simulations of ram pressure stripping where bright $\mathrm{H} \alpha$ emission is produced at the edges of dense neutral clouds (Tonnesen et al. 2011). For $\mathrm{f}_{\mathrm{H} \alpha} \approx 0.05$ (see, e.g., Section 8.4 in Jáchym et al. 2013), the upper limit on the amount of $\mathrm{H} \alpha$-emitting gas is about $1 \times 10^{8} \mathrm{M}_{\odot}$.

4. Spitzer observations revealed about $4 \times 10^{7} M_{\odot}$ of warm $(130-160 \mathrm{~K}) \mathrm{H}_{2}$ in the galaxy and the inner $20 \mathrm{kpc}$ of the tail (Sivanandam et al. 2010).

The total gas mass in the tail thus is $2 \times 10^{9} \lesssim \mathrm{M}_{\text {gas }} \lesssim$ $4 \times 10^{9} M_{\odot}$, including the ATCA H I upper limits, while the total gas mass in the disk of ESO 137-001 is $\sim 1 \times 10^{9} M_{\odot}$ (see Table 5). It means that the amounts of ram-pressure-stripped gas possibly exceed the current gas mass left in the disk. Since the original (pre-stripping) gas content of the galaxy estimated from its stellar mass and an assumption of a typical gas to stellar mass ratio is $\sim(0.5-1) \times 10^{10} M_{\odot}$ (Sun et al. 2010; Sivanandam et al. 2010), it also means that the observed amount of stripped gas nearly accounts for the missing gas from the disk. The mass ratio of the most abundant phases in the whole tail, the coldto-hot, $\left(\mathrm{H}_{2}+\mathrm{H}_{\mathrm{I}}\right)$-to-X-ray, gas spans from about $1: 1$ to $\lesssim 3: 1$, assuming the Galactic CO-to- $\mathrm{H}_{2}$ conversion factor and taking into account the H I upper limit. The amounts of cold and hot gas in the tail are thus large and similar.

Our observations show for the first time that $\mathrm{H}_{2}, \mathrm{H} \alpha$, and $\mathrm{X}$-ray emission can be at observable levels in a single rampressure-stripped tail at a large scale of several tens of kilo-

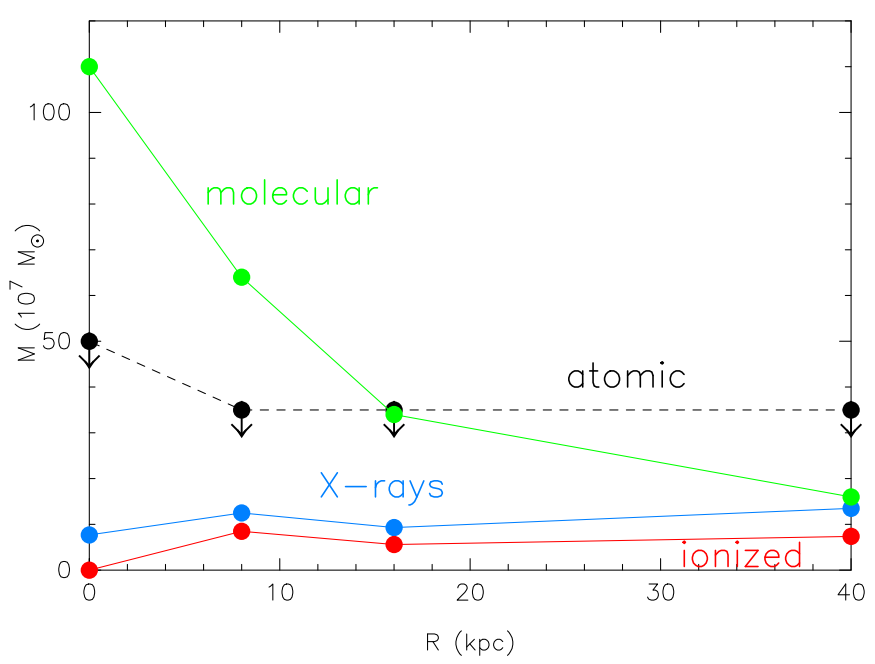

Figure 5. Masses of individual gas phases in the four observed regions (beams of $\sim 8 \mathrm{kpc}$ FWHM) as a function of projected distance from the galaxy-molecular gas (green), diffuse $\mathrm{H} \alpha$ (red), X-rays (blue), and upper limits on $\mathrm{H}$ I (black). A low $\mathrm{H} \alpha$ filling factor of 0.05 is used (see the text). Molecular gas mass depends on the correction factor to the Galactic value of the CO-to- $\mathrm{H}_{2}$ conversion factor. (A color version of this figure is available in the online journal.)

parsecs. This supports predictions of numerical simulations (Tonnesen et al. 2011; Tonnesen \& Bryan 2012) that further suggested that the amounts of X-ray-emitting gas and the starforming cold gas in the tail depend strongly on the surrounding ICM pressure. In their model with the thermal ICM pressure close to that at the location of ESO 137-001 (but with a lower ram pressure), the ratio of cold-to-hot components in the tail is about 1:1.4, and it drops to $\sim 1: 9$ for about a two times higher thermal pressure. Note, however, that in Tonnesen et al. (2011), the cold component corresponds to gas in the temperature range of $3 \times 10^{2}-10^{4} \mathrm{~K}$, and the hot component to $7 \times 10^{5}-4 \times 10^{7} \mathrm{~K}$, i.e., $0.06-3.45 \mathrm{keV}$, which is thus not completely consistent with the observations. They also estimated that in the Norma cluster the radius at which the ICM pressure falls below the minimum value needed for a tail to have observable X-ray emission is $\sim 250 \mathrm{kpc}$. This is very close to the projected distance of ESO 137-001 from the Norma center, which suggests that the galaxy may have lit up in soft X-rays rather recently.

\subsection{Systematic Gradient Along the Tail}

In order to understand the evolution of gas in the tail, it is of great interest to know how the balance between the different gas phases changes along the tail. It is expected that the rampressure-stripped cool gas either heats up by the surrounding hot ICM or cools via radiative cooling and transforms into molecular gas. In Table 5 and in Figure 5, we summarize the amounts of different gas phases in the observed tail regions (APEX beams) as a function of the projected distance from the disk. We note that the lines in Figure 5 connecting the four data points are intended to guide the eye and do not reflect real mass profiles between the observed positions. Figure 5 clearly shows that the farther from the disk, the amount of molecular gas decreases while the amounts of diffuse ionized and X-ray emitting components stay roughly constant. We summarize below the local mass ratios of individual gas phases along the tail.

1. The ratio of cold-to-hot $\left(\mathrm{H}_{2}\right.$-to-X-ray) masses decreases from about 5 in the 001-A region to $\sim 1$ in the $001-\mathrm{C}$ region, assuming a constant $\mathrm{CO}-\mathrm{to}-\mathrm{H}_{2}$ conversion factor. 
Table 6

The H $\alpha$ and X-Ray Fluxes and Masses in the Observed Regions

\begin{tabular}{lcrrrrr}
\hline \hline Source & $F_{0.5-2 \mathrm{keV}}$ & $M_{\mathrm{X}}$ & $F_{\mathrm{H} \alpha}$ & $F_{\mathrm{H} \alpha}^{\prime}$ & $M_{\mathrm{H} \alpha}$ & $\frac{F_{\mathrm{X}}}{F_{\mathrm{H} \alpha}^{\prime}}$ \\
\hline ESO 137-001 & 2.7 & 7.7 & 11.7 & $\ldots$ & $\ldots$ & $\ldots$ \\
001-A & 2.3 & 12.5 & 3.0 & 1.9 & 38 & 1.3 \\
001-B & 1.3 & 9.3 & 1.1 & 0.9 & 25 & 1.5 \\
001-C & 2.1 & 13.5 & 1.1 & 1.1 & 33 & 1.9 \\
\hline
\end{tabular}

Notes. The $\mathrm{H} \alpha$ and $\mathrm{X}$-ray fluxes and corresponding masses in individual observed points. Fluxes of the diffuse $\mathrm{H} \alpha$-emitting gas including the $\mathrm{H}$ II regions $\left(F_{\mathrm{H} \alpha}\right)$ and without the $\mathrm{H}$ II regions $\left(F_{\mathrm{H} \alpha}^{\prime}\right)$ are given. About $40 \%$ of the $\mathrm{H} \alpha$ diffuse flux in 001-A is from the galaxy and 001-B. The fluxes and masses are given in $10^{-14} \mathrm{erg} \mathrm{s}^{-1} \mathrm{~cm}^{-2}$, and $10^{7} f^{1 / 2} M_{\odot}$, respectively, where $f_{\mathrm{H} \alpha}$ and $f_{\mathrm{X}}$ represent the respective filling factor. The tail was approximated by a cylinder with a diameter of $4.8 \mathrm{kpc}$ (for 001-A and 001-B) and $5.5 \mathrm{kpc}$ (for 001-C).

2. The ratio of cool-to-warm ionized $\left(\mathrm{H}_{2}\right.$-to- $\left.\mathrm{H} \alpha\right)$ components along the tail decreases from $\sim 8$ in the $001-$ A region to $\sim 3$ in $001-\mathrm{C}$, assuming that the $\mathrm{H} \alpha$ volume-filling factor does not change.

3. The ratio of hot-to-warm ionized (X-ray-to-H $\alpha$ ) masses is quite similar in all three regions and close to $\sim 2$.

4. The warm-to-cold molecular gas ratio can be also estimated. The region defined in Sivanandam et al. (2010) as a"tail" covers about half of the 001-A pointing and a quarter of the $001-\mathrm{B}$ pointing. It contains $3.6 \times 10^{7} \mathrm{M}_{\odot}$ of warm $\mathrm{H}_{2}$, while $<4 \times 10^{8} M_{\odot}$ of cold $\mathrm{H}_{2}$. Thus, the warm-to-cold (mid-IR-to-CO) $\mathrm{H}_{2}$ mass fraction is $\gtrsim 0.1$.

The ratio of warm-to-cold molecular gas $>0.1$ in the inner tail of ESO 137-001 is higher than in star-forming galaxies, where it is usually 0.004-0.04 (Guillard et al. 2012; Nesvadba et al. 2010; Roussel et al. 2007). The observed value is close to ratios measured in cool cores of cluster and group central galaxies or in environments where molecular gas is shock- or cosmic-ray-heated rather than UV-heated. For example, in the intra-galactic medium of Stephan's Quintet the ratio of warmto-cold $\mathrm{H}_{2}$ was measured to $\approx 0.3$ by Guillard et al. (2012), who also suggested that a high heating rate in the $\mathrm{H}_{2}$ gas is probably maintained by turbulence invoked by galaxy-tidal arm collision. The observed value is consistent with shock heating (as suggested by Sivanandam et al. 2010) and possibly heat conduction from ICM as other sources of heat that must exist in the tail besides star formation. It is likely that the diffuse component of $\mathrm{H} \alpha$ in the tail is not associated with star formation but is powered by shocks or other processes, such as heat conduction (Sun et al. 2007). For example, shocks from ram pressure were proposed to be responsible for elevated levels of radio continuum emission, as well as for elevated ratios of warm $\mathrm{H}_{2} / \mathrm{PAH}$ in Virgo spirals being stripped (Murphy et al. 2009; Wong et al. 2014, respectively). Consequently, the CO emission in the tail of ESO 137-001 may not only trace cold molecular gas but also warmer gas (Guillard et al. 2012) and thus the actual amount of cold $\mathrm{H}_{2}$ in the gas-stripped tail could be lower than that corresponding to the standard CO-to- $\mathrm{H}_{2}$ conversion factor (e.g., Bolatto et al. 2013).

The $\mathrm{H} \alpha$ and X-ray fluxes and masses derived for the APEX apertures are listed in Table 6 . The $\mathrm{H} \alpha$ fluxes were derived using the case $\mathrm{B}$ recombination theory and we did not assume any intrinsic extinction for the diffuse $\mathrm{H} \alpha$ emission (although there may be as there is dust in the tail). A large uncertainty comes from the $\mathrm{H} \alpha$ emissivity and the filling factor. The X-ray masses are uncertain from the spectral model and the filling factor.
The positional coincidence of the $\mathrm{H} \alpha, \mathrm{X}$-ray, and CO emission in the tail of ESO 137-001 suggests that different gas components mutually interplay and possibly exchange energy. The origin of the observed soft X-ray emission in the tail is presumed to arise at the mixing interfaces of cold stripped ISM and the surrounding hot ICM (Sun et al. 2010; Tonnesen et al. 2011). The drop of molecular gas amounts along the tail could point to a decreasing mean gas density in the tail with increasing distance (for example, due to more acceleration at larger distances that makes the gas more spread out in the direction of the tail in its outer parts), to a long timescale of heating, or to a recent increase in the ram pressure stripping strength and stripping rate that has pushed more dense gas to the inner tail regions only (see Section 6). We note that our CO observations may be biased by targeting only the $\mathrm{H} \alpha$ peaks. Also, the changing X-factor along the tail could, to some extent, contribute to the decrease of molecular gas. Even so, in ESO 137-001, it is the first time that we can follow in rough outline the distribution of cold molecular gas in a ram-pressure-stripped tail.

\section{RAM PRESSURE STRIPPING OF ESO 137-001}

The previous thorough analysis of Sivanandam et al. (2010); Sun et al. $(2007,2010)$ showed that ESO 137-001 is a clear case of ram pressure stripping, which is consistent with its high H I-deficiency $>10$, the undisturbed morphology of its stellar disk, and the presence of a prominent one-sided tail. Other indicators suggest that the effects of ram pressure on the galaxy may have been strong: ESO 137-001 is projected very close to the center of the Norma cluster which, moreover, is a rich and massive cluster. Thus, the local ICM density is presumably high, as well as the galaxy's orbital velocity. In addition, the rather low mass of ESO 137-001 makes it easier to strip. Its rotation velocity of $\sim 110 \mathrm{~km} \mathrm{~s}^{-1}$ is lower than the rotation velocities of the most studied Virgo ram-pressure-stripped galaxies with typically $v_{\text {rot }} \sim 130 \mathrm{~km} \mathrm{~s}^{-1}$ (NGC 4522, NGC 4402; Kenney \& Koopmann 1999; Crowl et al. 2005) up to $\sim 170 \mathrm{~km} \mathrm{~s}^{-1}$ (NGC 4330; Abramson et al. 2011).

\subsection{Possible Orbits}

In order to estimate the ram pressure stripping history of ESO 137-001, we will first numerically study its possible orbits in the Norma cluster. Such orbits need to be consistent with the galaxy's current position on the sky, the LOS velocity, and the plane of the sky velocity direction (indicated by the projected tail direction). ${ }^{12}$ The free parameters of the model are the current LOS distance relative to the cluster center and velocity component in the plane of the sky. According to the measured absence of a radial velocity gradient along the tail, most of ESO 137-001's orbital motion must be occurring close to the plane of the sky, and the actual distance of the galaxy from the cluster center is thus possibly close to the projected one. The orientation of the gas-stripped tail pointing away from the cluster center then suggests that the galaxy is currently approaching the pericenter.

The gravitational potential of the Norma cluster can be modeled with a Navarro-Frenk-White profile (NFW; Navarro et al. 1996) with the parameters given in Table 7 . This yields a cluster with $M_{\text {vir }}=1.1 \times 10^{15} M_{\odot}$ and $R_{\text {vir }}=2.2 \mathrm{Mpc}$ and a

\footnotetext{
12 The position angle of the (main) tail varies along its length-in the inner parts, both young stellar streams in the HST image and the X-ray emission show P.A. $\sim 316^{\circ}$, while in the outer tail it is $\sim 306^{\circ}$. We measure the P.A. from north to west.
} 
Table 7

Parameters of the Cluster and Galaxy Models

\begin{tabular}{lcc}
\hline \hline Cluster: & $r_{\mathrm{s}}=346 \mathrm{kpc}$ & $c=6.2$ \\
& $\rho_{\mathrm{ICM}, 0}=4.82 \times 10^{-24} \mathrm{~kg} \mathrm{~m}^{-3}$ & $r_{c}=184.5 \mathrm{kpc}$ \\
& $\beta=0.555$ & \\
\hline Galaxy: & $M_{\mathrm{d}}=1 \times 10^{10} M_{\odot}$ & $a_{\mathrm{d}}=3 \mathrm{kpc}^{\mathrm{a}}$ \\
& $M_{\mathrm{b}}=1 \times 10^{9} M_{\odot}$ & $a_{\mathrm{b}}=0.5 \mathrm{kpc}$ \\
& $M_{\mathrm{h}}=7 \times 10^{10} M_{\odot}$ & $a_{\mathrm{h}}=10 \mathrm{kpc}$ \\
\hline
\end{tabular}

Notes. Parameters of the cluster and galaxy models used for semi-analytic calculations of ram pressure stripping. Cluster parameters are from Sivanandam et al. (2010).

a Measured by Sun et al. (2007)

distribution of mass with radius that is well consistent with the values measured by Woudt et al. (2008). Since the Norma cluster is not relaxed, its dark matter distribution may be bimodal in reality, following the two interacting subclusters around giant ellipticals ESO 137-008 and ESO 137-006 (see Figure 1). ESO 137-001, however, lies outside of the central merging region. Also the distribution of ICM in the NW part of the cluster looks more symmetric than the opposite side where the X-ray emission is elongated. In our modeling, ESO 137-006 was considered to be the cluster center (see Figure 1) since the peak of the galaxy density distribution is located only $\sim 3^{\prime}$ from it (Woudt et al. 2008).

We cover a wide range of values of the free parameters: the velocity component $v_{x y}$ in the plane of the sky spans from $1000 \mathrm{~km} \mathrm{~s}^{-1}$ to $4000 \mathrm{~km} \mathrm{~s}^{-1}$ and the LOS distance $z$ relative to the cluster center from $-600 \mathrm{kpc}$ to $600 \mathrm{kpc}$ (although negative and positive distances are almost equivalent due to only a small radial velocity component). For a given velocity, the trend is as follows: with an increasing LOS distance $z$, the pericenter distance grows and the apo-to-pericenter ratio decreases. Cosmological simulations (e.g., Wetzel 2011) have suggested that galaxies at $z=0$ infalling to massive $(M=$ $10^{14}-10^{15} M_{\odot}$ ) host halos occur preferentially on radial orbits with an average eccentricity of $e=0.85$, and thus a mean apo-to-pericentric ratio $r_{\text {apo }} / r_{\text {peri }}=(1+e) /(1-e)$ of $\sim 12$. Mean pericenter and apocenter distances are $r_{\text {peri }}=0.13 R_{\mathrm{vir}}$ and $r_{\text {apo }}=1.6 R_{\mathrm{vir}}$, respectively. Typical turnaround radii (where the characteristic infall velocity is equal to the Hubble flow) of radially infalling galaxies in clusters are thus several megaparsecs (e.g., Zu \& Weinberg 2013). ESO 137-001 is believed to be on its first infall.

Although the velocity dispersion of $\sim 949 \mathrm{~km} \mathrm{~s}^{-1}$ measured within a $\sim 0.67 \mathrm{Mpc}$ radius of the Norma cluster (Woudt et al. 2008 ) suggests a $3 \mathrm{D}$ orbital velocity of $\sqrt{3} \sigma=1644 \mathrm{~km} \mathrm{~s}^{-1}$, all our model runs for ESO 137-001 with velocities lower than $\sim 3000 \mathrm{~km} \mathrm{~s}^{-1}$ are too compact and do not cross the cluster virial radius. This is illustrated in Figure 6, where model orbits with the current plane of sky velocity component in the range from $2100 \mathrm{~km} \mathrm{~s}^{-1}$ to $3900 \mathrm{~km} \mathrm{~s}^{-1}$ are shown. Thus, to reach the above typical orbital parameters of infalling galaxies in cosmological simulations, our modeling suggests that ESO 137001 has a rather high orbital velocity $\gtrsim 3000 \mathrm{~km} \mathrm{~s}^{-1}$. This is also supported by the simulations of Tonnesen \& Bryan (2008), which suggested that the relative galaxy-ICM velocities within the inner $\sim 300 \mathrm{kpc}$ of a large $M_{200}=6 \times 10^{14} M_{\odot}$ cluster are larger than $\sim 2500 \mathrm{~km} \mathrm{~s}^{-1}$.

As a fiducial orbit for further ram pressure modeling, we select the one with $v=3300 \mathrm{~km} \mathrm{~s}^{-1}$ and the LOS distance relative to the cluster center of $z=300 \mathrm{kpc}$ (see the dashed curve
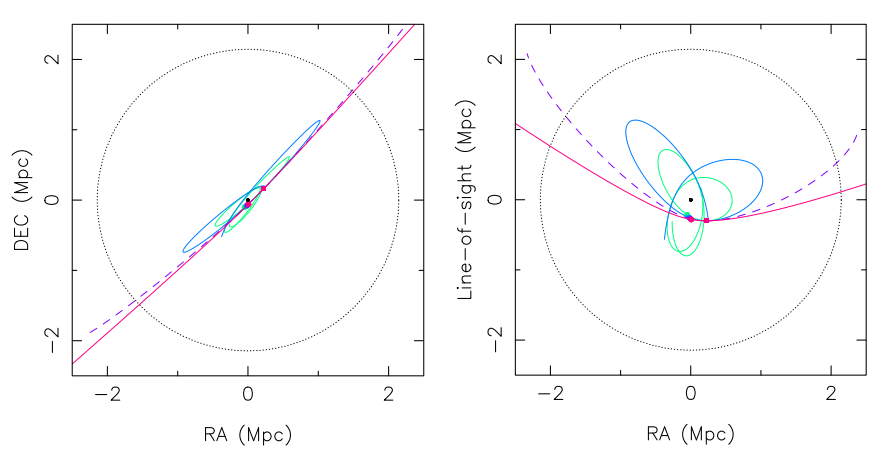

Figure 6. R.A.-Decl. and R.A.-LOS views of the model orbits with the plane of sky velocity component changing from $2100 \mathrm{~km} \mathrm{~s}^{-1}$ to $3900 \mathrm{~km} \mathrm{~s}^{-1}$, with a $600 \mathrm{~km} \mathrm{~s}^{-1}$ step, and the LOS distance of the galaxy of $300 \mathrm{kpc}$ relative to the cluster center. The cluster virial radius of $\sim 2.2 \mathrm{Mpc}$ is shown by the dashed circle. The sections of orbits shown correspond to a travel time of $\sim 5.5 \mathrm{Gyr}$. The square marks the current position of the galaxy and the dots mark the pericenters. The $3300 \mathrm{~km} \mathrm{~s}^{-1}$ (dashed) orbit is our fiducial orbit used to model ram pressure stripping.

(A color version of this figure is available in the online journal.)

in Figure 6) for which the pericenter distance and the peri-toapocenter ratio are consistent with the mean values predicted for infalling galaxies from cosmological simulations. Along the fiducial orbit the galaxy is currently about 100 Myr before pericenter.

\subsection{Effects of Ram Pressure}

We will follow the effects of ram pressure on ESO 137-001 along the fiducial model orbit. By means of semi-analytic calculations, we estimate how ISM parcels with different column densities react to a time varying ram pressure. We thus neglect all hydrodynamic effects, such as the Kelvin-Helmholtz (KH) and Rayleigh-Taylor (RT) instabilities or gas compression, that can accompany the dynamical ram pressure. The acceleration due to local ram pressure may be expressed as

$$
\frac{d v}{d t} \Sigma_{\mathrm{ISM}}=\rho_{\mathrm{ICM}}\left(v-v_{0}\right)^{2}-\frac{\partial \Phi}{\partial z} \Sigma_{\mathrm{ISM}},
$$

where $\Sigma_{\text {ISM }}$ is the mean column density of an ISM parcel, $\left(v-v_{0}\right)$ is the vertical component of its relative velocity with respect to the surrounding ICM, and $(\partial \Phi / \partial z) \Sigma_{\text {ISM }}$ is the gravitational restoring force of the disk + bulge + halo galaxy components in the vertical direction. We then numerically solve the equation of motion (Equation (2)) along the fiducial orbit for different values of $\Sigma_{\text {ISM }}$ and for different disk radii. We have not accounted for the changing wind angle along the orbit and instead use a face-on wind. ${ }^{13}$

To model the ICM distribution, we follow Boehringer et al. (1996), who fitted the western, less disturbed part of the A3627 cluster with a $\beta$-model centered on the X-ray peak located about 5'.5 west of ESO 137-006. The $\beta$-profile parameters are given in Table 7 , together with the parameters of the model galaxy. For ESO 137-001, the $K$-band velocity-luminosity relation $M_{K}=-22.5 \mathrm{mag}(\mathrm{NED})$ yields a rotation velocity of $\sim 110-120 \mathrm{~km} \mathrm{~s}^{-1}$ (Courteau et al. 2007). Assuming a flat rotation curve, this corresponds to a dynamical mass of $\sim 3.5 \times 10^{10} M_{\odot}$ within the observed stellar disk radius of $\sim 40^{\prime \prime} \cong 13 \mathrm{kpc}$ (Sivanandam et al. 2010). We assume a simple Plummer dark matter halo with the total mass of $\sim 7 \times 10^{10} M_{\odot}$ and a scale radius of $10 \mathrm{kpc}$, although the halo parameters

\footnotetext{
13 With the small relative radial velocity of the galaxy and its high inclination, ram pressure is expected to be operating on the galaxy close to face-on $\left(\lesssim 30^{\circ}\right)$.
} 


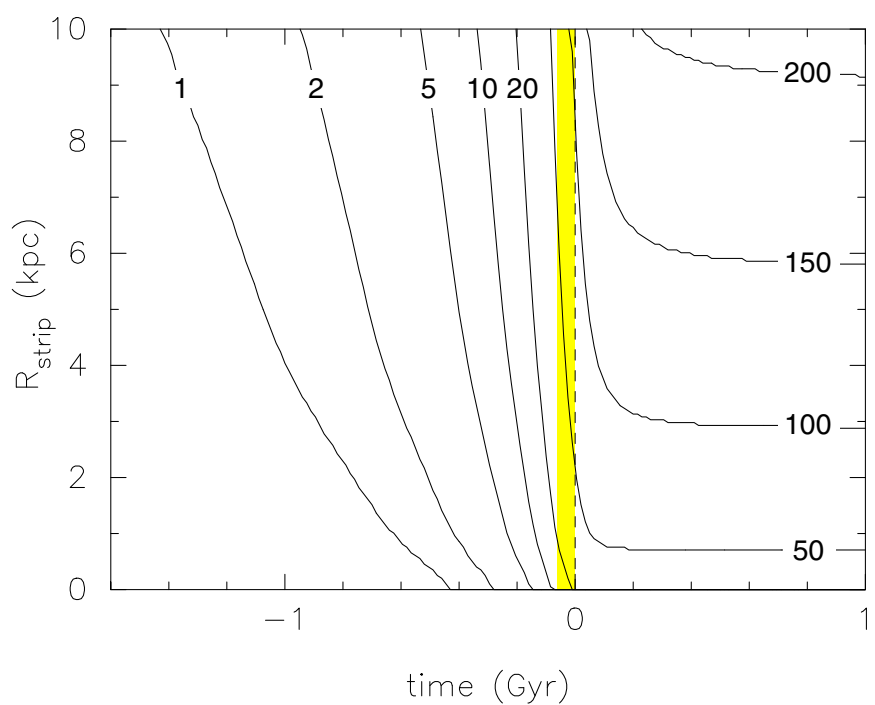

Figure 7. Effects of ram pressure along the fiducial orbit - evolution of stripping radii of ISM components with different column densities (curves from left to right; values are in $M_{\odot} \mathrm{pc}^{-2}$ units). The peak of ram pressure occurs at $0 \mathrm{Gyr}$ (dashed vertical line). Time remaining to the peak is indicated with the shaded area

(A color version of this figure is available in the online journal.)

are somewhat uncertain and the simulation is sensitive to the parameters.

Along our fiducial orbit, the galaxy is currently about $100 \mathrm{Myr}$ before pericenter, but, due to the offset of ICM distribution from ESO 137-006, it is only about $60 \mathrm{Myr}$ before peak ram pressure. Correspondingly, the current ram pressure has a high value of $\sim 1.5 \times 10^{-10}$ dyne $\mathrm{cm}^{-2}$ and the peak ram pressure will reach $\sim 2.1 \times 10^{-10}$ dyne $\mathrm{cm}^{-2}$. The FWHM of the ram pressure time profile is $\sim 200 \mathrm{Myr}$.

Figure 7 displays the evolution of stripping radii for ISM components with different column densities along the fiducial orbit. We consider an ISM element to be stripped when its vertical velocity exceeds the escape speed from the galaxy. Figure 7 suggests that gas with $\Sigma_{\text {ISM }} \lesssim 10 M_{\odot} \mathrm{pc}^{-2}$ is currently completely stripped from the galaxy $\left(R_{\text {strip }}=0 \mathrm{kpc}\right)$, and that stripping has proceeded to denser gas (about 20-50 $M_{\odot} \mathrm{pc}^{-2}$ ) at larger disk radii. The figure also indicates the timescale of stripping. As expected, lower-density gas begins to be accelerated earlier along the orbit than more dense clumps, and stripping also proceeds from outer radii of the disk inward. It means that a mixture of different density gas forms in the tail since denser clumps from outer radii may be stripped to similar distances at the same time as less dense gas from inner radii. The timescale for stripping depends on the ISM parcel's column density and on its location in the disk. In Figure 7, one can also infer how dense ISM will be stripped from the galaxy in the future after it passes through the peak ram pressure.

To compare the predictions of our model with the observed length of the tail of ESO 137-001, Figure 8 displays the vertical distances that ISM elements have reached as a function of their column density and radius in the disk (solid curves). It indicates that gas with the maximum density of $\sim 20 M_{\odot} \mathrm{pc}^{-2}$ (depending on radius) could currently be stripped to large distances exceeding the observed length of ESO 137-001's $\mathrm{X}$-ray tail, while denser gas of up to $\sim 80-100 \mathrm{M}_{\odot} \mathrm{pc}^{-2}$ is only pushed to inner tail regions. More specifically, Table 8 gives the column densities of elements stripped from different disk radii to vertical distances matching those of the whole tail length, and

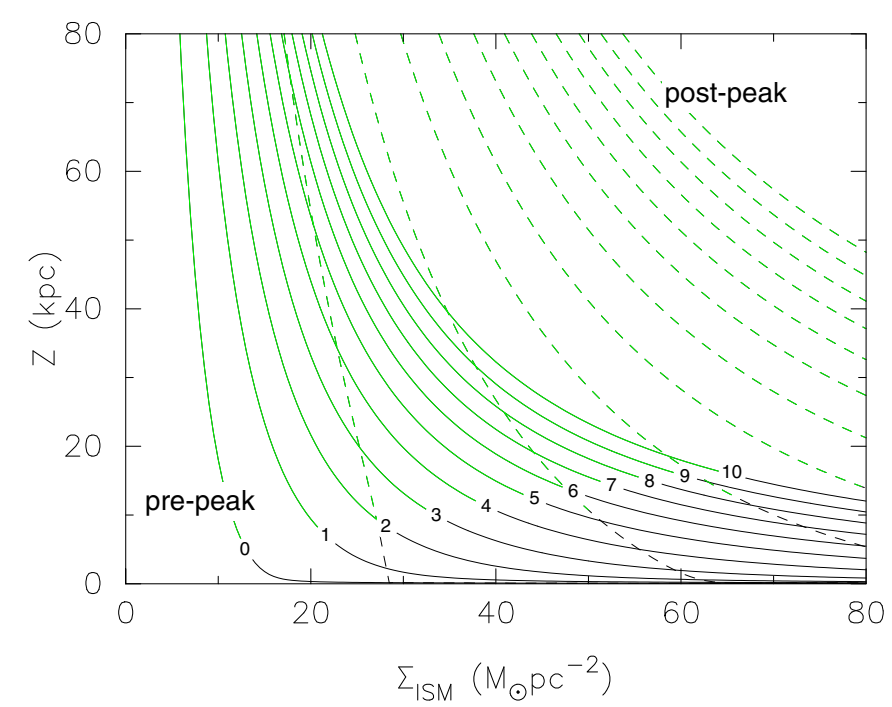

Figure 8. Effects of ram pressure along the fiducial orbit-vertical distances which can obtain ISM elements with different column densities from different disk radii (0-10 kpc; curves from left to right; see labels in kiloparsecs) at present, $\sim 60$ Myr pre-peak (solid lines), and, for comparison, symmetrically at $\sim 60$ Myr post-peak (dashed lines). The radius labels separate the segments of the curves where ISM is stripped (green) or only pushed but still bound to the galaxy.

(A color version of this figure is available in the online journal.)

Table 8

Column Densities of Stripped ISM in Our Model

\begin{tabular}{lccc}
\hline \hline Vertical Distance & \multicolumn{3}{c}{ Radius in the Disk } \\
\cline { 2 - 4 } & $2 \mathrm{kpc}$ & $6 \mathrm{kpc}$ & $10 \mathrm{kpc}$ \\
\hline tail $(80 \mathrm{kpc})$ & 10 & 17 & 20 \\
$001-\mathrm{C}(40 \mathrm{kpc})$ & 15 & 25 & 35 \\
$001-\mathrm{A}(10 \mathrm{kpc})$ & 25 & 60 & 90 \\
\hline
\end{tabular}

Notes. Approximate column densities (in $M_{\odot} \mathrm{pc}^{-2}$ units) of ISM stripped (or pushed) along our fiducial orbit from different disk radii to distinct vertical distances for the observed tail of ESO 137-001. The results follow from Figure 8.

of the projected locations of the 001-C and 001-A regions. For comparison, a symmetrical, 60 Myr post-peak situation, i.e., about 120 Myr from now, is also shown in Figure 8 (dashed curves).

Our calculations show the effects of ram pressure on ISM with different column densities and suggest that rather dense clumps may be stripped/shifted from the disk of ESO 137-001. However, real GMCs have strong internal density gradients, and ram pressure is thus most likely able to ablate the outer portions of a cloud rather than remove it entirely (even though the cloud's average density may be low enough to be displaced, according to the model). Current observations of Galactic GMCs have suggested that column densities of dense cores of GMCs reach values of several hundreds of $M_{\odot} \mathrm{pc}^{-2}$ while the mean column density over the whole clouds is rather low $\left(\sim 42 M_{\odot} \mathrm{pc}^{-2}\right.$; Heyer et al. 2009), much lower than previously estimated by Solomon et al. (1987). Figure 7 shows that ram pressure has likely been increasing substantially during the last couple hundred million years and thus it is possible that the ablation of clouds may have progressed into the central parts of some of them and effectively stripped them.

The exceptional features of ESO 137-001 (in comparison with most other known RPS galaxies), such as the bright and long X-ray tail, unprecedented SF activity downstream from 
the galaxy, as well as the wealth of CO emission in the tail, support the idea of recent strong ram pressure stripping. This is also suggested by the presence of dust filaments revealed in HST and Herschel imaging since typical column densities of the dust clouds that produce significant optical extinction are at least $\sim 5 M_{\odot} \mathrm{pc}^{-2}$ (Abramson \& Kenney 2014). Very recently, Ebeling et al. (2014) reported a discovery of several strongly ram-pressure-stripped galaxies falling into massive $z>0.3$ clusters. Similarly to ESO 137-001, these galaxies are located close ( $\sim 90-360 \mathrm{kpc}$ in projection) to the center of their host cluster. It is thus also possible that in these galaxies, ram pressure stripping may have currently proceeded to denser ISM since $\mathrm{H}$ I removal is expected to take place at larger distances from the cluster center along the orbit of the infalling galaxies.

\section{DISCUSSION}

In the following sections, we will identify possible traces of turbulence in the tail of ESO 137-001 from the kinematic structure of the detected $\mathrm{CO}$ lines, and we will discuss turbulence as a possible mechanism suppressing star formation in the stripped gas. Since hydrostatic pressure in galactic disks is suggested to be the key parameter that determines the transformation between atomic and molecular gas, we will compare the ICM pressure operating on ESO 137-001 with values typical in galaxies. Further, we will discuss possible origins of the detected molecular gas in the galaxy's tail, as well as think about the evolutionary state of the "orphan" H II regions occurring in the broad parts of the tail.

\subsection{Multiple Line Components and Tail Kinematics}

While the ESO 137-001 main body CO line is only slightly asymmetric, the tail spectral lines all show substructure. Using the CLASS routine FIT, we decomposed the spectra in the 001-A and 001-B positions into several Gaussian components (see Figure 3 and Table 3), showing that the kinematics of the $\mathrm{CO}$-emitting gas is rather complex, especially in the inner parts of the tail. This may reflect individual (large) condensations of dense gas possibly consistent with the observed distinct H II regions (as already suggested in Figure 3 by blue bars showing radial velocities of the $\mathrm{H}$ II regions). The molecular gas masses of the individual resolved components span a range of $\sim 5 \times 10^{7}-4 \times 10^{8} M_{\odot}$. The FWHMs of their Gaussian fits are of the order of $\sim 25-64 \mathrm{~km} \mathrm{~s}^{-1}$, i.e., $\sim 11-27 \mathrm{~km} \mathrm{~s}^{-1}$ velocity dispersions (since the relationship between the FWHM of a Gaussian fit and the velocity dispersion is FWHM $\approx$ $2.3548 \sigma)$. These rather small values are consistent with the ongoing star formation in the spatially associated regions (star clusters) since the gas is more concentrated and possibly self-gravitating. Much better spatial and spectral resolution would be needed to further resolve smaller entities, such as individual molecular clouds whose velocity dispersions are smaller, typically $\sim 10 \mathrm{~km} \mathrm{~s}^{-1}$, and whose masses and diameters are $10^{5}-10^{6} M_{\odot}$ and $10-100 \mathrm{pc}$, respectively (e.g., Solomon et al. 1987).

In the observed tail regions, the $\mathrm{CO}$ emission is more extended in velocity than in the main body (see Figure 3 and Table 3), especially in the 001-B pointing where individual line components are clearly distinct. While some of the velocity spread could be due to the rotational motion of the disk that will survive into the (inner) tail, we expect turbulent motions driven to the stripped ISM by the high-speed interaction between the galaxy and the surrounding ICM to be important. A turbulent velocity field was observed to develop in the stripped gas in numerical simulations due to the $\mathrm{KH}$ instability that leads to the formation of eddy-like structures in the tail (e.g., Tonnesen \& Bryan 2010; Roediger \& Hensler 2005; Roediger et al. 2006). The typical rms turbulent velocity averaged over a galaxy's tail is predicted to be of the order of $\sim 100 \mathrm{~km} \mathrm{~s}^{-1}$ (e.g., Subramanian et al. 2006). For example, in the Virgo cluster galaxy NGC 4388, Yoshida et al. (2004) revealed the turbulent kinematic structure of the ram-pressure-stripped ionized gas with abrupt velocity changes of up to $\sim 300 \mathrm{~km} \mathrm{~s}^{-1}$. The dense gas that we detect in CO line emission possibly does not reflect the whole range of turbulent motions present in the tail of ESO 137-001.

The present observations allow us to roughly estimate the turbulent kinetic energy carried by the $\mathrm{CO}$-emitting gas in the tail of ESO 137-001. From $E_{\text {kin }}=3 / 2 M_{\mathrm{H}_{2}} \sigma_{\mathrm{CO}}^{2}$, where $M_{\mathrm{H}_{2}}$ is the molecular gas mass derived from the $\mathrm{CO}(2-1)$ intensity and $\sigma_{\mathrm{CO}}$ is the one-dimensional, LOS velocity dispersion measured from the (multiple) Gaussian fits of the $\mathrm{CO}(2-1)$ lines (see Table 3), the kinetic energies for the 001-A, 001-B, and 001-C tail positions are about $11.6 \times 10^{54} \mathrm{erg}, 5.5 \times 10^{54} \mathrm{erg}$, and $7.4 \times 10^{54} \mathrm{erg}$, respectively. In total, the observed CO-emitting gas in the tail carries $\sim 2.5 \times 10^{55} \mathrm{erg}$. For comparison, the main body position has a kinetic energy of about $3.8 \times 10^{55} \mathrm{erg}$, however, most of its line width comes from (ordered) rotational motion, not turbulent motion. This is also partly true for the tail positions, as suggested from radial velocities of the $\mathrm{H}$ II regions (Sun et al. 2010). The total turbulent energy in the CO-emitting gas in the tail thus may be lower than the above estimated value by a factor of few. For comparison, we can estimate the thermal energy of the hot ( $\sim 0.8 \mathrm{keV}$; Sun et al. 2010) X-ray-emitting plasma encompassed in the three observed regions in the tail of ESO 137-001 from $E_{\text {therm }}=3 / 2 n_{e} k T$. Assuming the beam area $\sim 80 \mathrm{kpc}^{2}$, the tail LOS width $\sim 7.8 \mathrm{kpc}$, and the electron density $n_{e} \sim 0.007 \mathrm{~cm}^{-3}$ (Sun et al. 2010), the carried thermal energy is $\sim 7 \times 10^{56} \mathrm{erg}$. Despite the limitations of the estimate, it suggests that the thermal energy of the hot gas is larger by a factor of $\sim 10$ than the turbulent energy of the CO-emitting gas.

\subsection{Molecular Gas Fraction versus Local Pressure in the Tail}

In galaxies, the molecular-to-atomic surface density ratio $\left(R_{\mathrm{H}_{2}}=\Sigma_{\mathrm{H}_{2}} / \Sigma_{\mathrm{HI}}\right)$ correlates with the interstellar pressure in galaxies (Blitz \& Rosolowsky 2004, 2006). In Figure 9, we compare the lower limits on $R_{\mathrm{H}_{2}}$ measured in the observed regions of ESO 137-001 (corresponding to our APEX detections and the ATCA upper limits; see Table 5) with data from disks of nearby galaxies (Blitz \& Rosolowsky 2006; Leroy et al. 2008) in a figure adopted from Krumholz et al. (2009). In the figure, we further constrain the thermal and total (thermal + ram) ICM pressure operating on ESO 137-001, corresponding to X-ray Chandra observations (Sun et al. 2010) and our simulations (Section 6). The surrounding ICM thermal pressure at the position of ESO 137-001 in A3627 is $\sim 1.8 \times 10^{-11}$ dyne $\mathrm{cm}^{-2}=1.3 \times 10^{5} k_{\mathrm{B}} \mathrm{K} \mathrm{cm}^{-3}$. The resulting area in Figure 9 is filled with a color gradient, indicating that if actual values of $\Sigma_{\mathrm{HI}}$ were known, the lower limits on $R_{\mathrm{H}_{2}}$ in ESO 137-001 would very likely shift to higher values and thus would cover values observed in disks of nearby galaxies.

Figure 9 shows that the ICM (thermal and ram) pressure at the location of ESO 137-001 in the Norma cluster is similar to pressures operating in completely different environments of galactic disks. This offers an explanation for the detected bulk of molecular gas in the gas-stripped tail of the galaxy. For comparison, in Figure 9 we also indicate the value of the thermal 


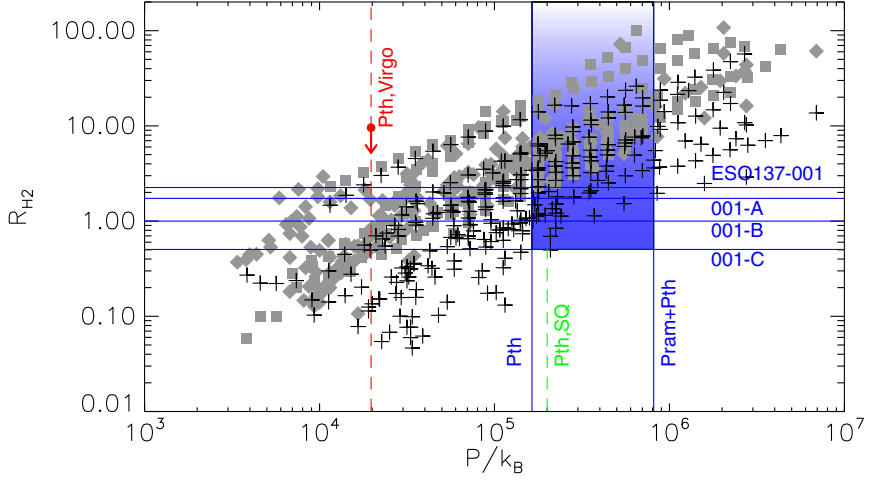

Figure 9. Molecular-to-atomic ratio, $R_{\mathrm{H}_{2}}=\Sigma_{\mathrm{H}_{2}} / \Sigma_{\mathrm{HI}}$, as a function of midplane pressure (in $\mathrm{K} \mathrm{cm}^{-3}$ ) in observations of nearby galaxies (squares-Blitz \& Rosolowsky 2006; diamonds-Leroy et al. 2008) and theoretical predictions of Krumholz et al. (2009, plus signs). Lower limits on $R_{\mathrm{H}_{2}}$ in the observed regions of ESO 137-001 are shown, together with values of thermal and total (thermal + ram) ICM pressure on the galaxy. For comparison, values of thermal pressure of the ICM in the Virgo cluster (at the position of IC3418 galaxy; red), and of the hot intra-group plasma in the densest, X-ray-brightest region in the Stephan's Quintet (green) are indicated (Jáchym et al. 2013; Guillard et al. 2009). Image adapted from Krumholz et al. (2009).

(A color version of this figure is available in the online journal.)

pressure in the Virgo cluster, at the location of the ram-pressurestripped dwarf galaxy IC3418. It is about an order of magnitude lower than the thermal pressure at ESO 137-001, thus suggesting much lower $R_{\mathrm{H}_{2}}$ ratios. Consistently, an upper limit on $R_{\mathrm{H}_{2}}$ in one of the observed regions in the outer tail of IC3418, where $\mathrm{CO}$ was only measured to upper limits and $\mathrm{H}$ i was detected (Jáchym et al. 2013; Kenney et al. 2014), is shown with the red arrow. Further, in Figure 9, the value of the thermal pressure of the hot plasma in the densest star-forming inter-galactic region in the X-ray brightest area ("ridge") of Stephan's Quintet is also indicated (Guillard et al. 2009). We note that the average thermal pressure in the Stephan's Quintet compact group is lower than the pressures in the Norma and Virgo clusters.

Although we find that the molecular-to-atomic gas ratios in the gas-stripped tail of ESO 137-001 may be consistent with values typical in galactic disks due to similar pressures in the two environments, SFE in the tail is much lower than in the galaxies (as shown in Section 4.2). Among the effects preventing star formation could be large-scale turbulence driven from interaction with the surrounding ICM. Turbulence can provide further support against gravity in cold molecular clouds, leading to small SFEs. Sivanandam et al. (2010) detected pure rotational $\mathrm{H}_{2}$ transitions $\mathrm{S}(0)-\mathrm{S}(7)$ in the inner tail of ESO 137-001, suggesting that during momentum transfer by ram pressure, shocks are indeed driven into the ISM, which increases its thermal and turbulent pressure. Moreover, they showed that $\mathrm{H}_{2} \mathrm{~S}(1)$ emission is (in the inner tail) much more extended than $8 \mu \mathrm{m}$ and $24 \mu \mathrm{m}$ emission, indicating that star formation proceeds only in a part of the stripped molecular gas. This may suggest that most of the molecular gas detected in the stripping tail of ESO 137-001 is most likely not self-gravitating due to turbulence and survives due to a lack of photodissociating UV radiation. Also, magnetic fields could prevent the collapse of molecular clouds.

\subsection{Origin of $\mathrm{H}_{2}$ in the Tail}

In this section, we qualitatively discuss the origin of molecular gas in the tail of ESO 137-001. The total $\mathrm{H}_{2}$ mass of more than $10^{9} M_{\odot}$ revealed within the three APEX apertures in the gas- stripped tail of the galaxy is a large amount possibly exceeding the original $\mathrm{H}_{2}$ mass in the galaxy. From the scaling relation deduced, for example, from the COLDGASS survey (Saintonge et al. 2011) or from a typical molecular gas fraction of $\sim 0.1$ in an Sc galaxy (Obreschkow \& Rawlings 2009), an average $\mathrm{H}_{2}$ mass of $\sim(0.5-1) \times 10^{9} M_{\odot}$ is expected in an unperturbed galaxy with a stellar mass of $\sim 10^{10} M_{\odot}$, although such estimates are only approximate (within a factor of few).

The origin of the molecular gas detected in the tail may be twofold. Either it has entirely formed in situ out of stripped atomic gas, or some pre-existing molecular gas contributed to it. For the in-situ formation scenario, the density of the stripped gas plays a role since it determines the timescale for condensation and $\mathrm{H}_{2}$ formation following an inverse relation (Guillard et al. 2009). Higher-density clumps can then radiatively cool down more easily and eventually form molecular gas, while the lowdensity stripped phase is compressed by the ICM, starts to mix with it, and likely accounts for the X-ray emitting hot gas in the tail (Tonnesen et al. 2011). Our simplified calculations in Section 6 suggested that ram pressure operating on ESO 137-001 has been strong enough to shift (or even strip) ISM clumps with column densities exceeding values typical in some molecular clouds. Since stripping proceeds (for a given disk radius) from low- to high-density gas, distant tail regions are more likely to host $\mathrm{H}_{2}$ that formed in situ from a lower-density gas phase, while inner tail may contain also directly stripped dense, possibly molecular ISM clumps.

The question arises whether the molecular phase can survive the effects of ram pressure and thus directly contribute to the observed CO surface brightness. Molecular clouds are affected in several ways - photodissociated by the UV radiation, X-ray dissociated (as in X-ray dissociation regions, XDRs; Maloney et al. 1996), disrupted and ablated by hydrodynamic effects connected to the ram pressure stripping, or dissolved due to their limited lifetime that typically is $\sim$ few $\times 10^{7} \mathrm{yr}$ (e.g., Murray 2011). However, it can be shown that the timescales of the hydrodynamic processes are long and may be suppressed by magnetic fields (Yamagami \& Fujita 2011), and that UV radiation, which is the dominant removal process in the stellar disks, is presumably absent in the tail environment. Numerical simulations also suggested that high ram pressures compress ISM clouds, making them more resistant to ablation (Tonnesen \& Bryan 2009). Consequently, it is possible that molecular clouds could survive through at least $\sim 10^{8} \mathrm{yr}$ and indeed get by ram pressure to the (inner) tail. Sivanandam et al. (2010) also suggested that the original molecular phase in the disk may be dissociated by the shock induced by the supersonic ram pressure. Such a shock-dissociated molecular gas, however, is expected to reform in the tail if dust survives (e.g., Guillard et al. 2009).

The presence of dust is crucial for $\mathrm{H}_{2}$ formation. The HST (see Figure 2, right panel; M. Sun et al., in preparation) and Herschel SPIRE (S. Sivanandam et al., in preparation) imaging indeed revealed in ESO 137-001 a dust trail emanating from the galaxy that is coaligned with its gas-stripped tail up to $\sim 40 \mathrm{kpc}$. In summary, we suggest that the two scenarios of the origin of $\mathrm{H}_{2}$ combine in the gas-stripped tail of ESO 137-001. The wealth of $\mathrm{CO}$ emission observed in its inner tail may correspond to directly stripped, relatively dense ISM clumps that not only are able to quickly form $\mathrm{H}_{2}$, but also may possess a molecular phase that survived the effects of ram pressure. The $\mathrm{CO}$ emission detected in the outer tail region is then more likely to originate from in situ formation from a stripped diffuse atomic component. Some $\mathrm{H}$ I thus must be present in the tail of ESO 137-001. It is possible 
that the ATCA observations (Vollmer et al. 2001) are at the limit of detection. Data analysis of deeper observations is ongoing with ATCA (M. Sun et al., in preparation).

\subsection{Orhpan H II Regions in the Broad Tail}

We have not observed the broad parts of the tail where "orphan" H II regions not associated with any X-ray emission occur (see the left panel of Figure 2). However, their $\mathrm{H} \alpha$ luminosities are comparable to those in the central tail, suggesting that a bulk of the $\mathrm{CO}$ emission could also be present in the broad tail. Calculations in Section 6 suggest that these regions were formed from relatively dense gas that was stripped from the outer disk $\sim($ few $\times) 10^{8}$ yr earlier than equally dense gas from the inner disk. This is due to the gravitational restoring force that is weaker at larger galactocentric radii and thus a weaker ram pressure is required to strip gas of a given surface density from the outer disk than from the inner disk. However, all $\mathrm{H}$ II regions that are spread through the tail are of a similar age $(<10 \mathrm{Myr})$. This suggests that star-forming $\mathrm{H}$ II regions are constantly forming and dying away throughout the tail, possibly due to the evolution of the stripped gas. This is also seen in numerical simulations where the exact temperature and density of the stripped gas determines how long it takes before the gas cools and condenses into clouds, invoking a large spread in the height of stars above the disk (Tonnesen \& Bryan 2012). It is not clear why X-ray emission is absent in the broad tail. It may be due to too much mixing that already lowered the density of the hot gas to the point where the X-ray emissivity is low (Tonnesen et al. 2011). The orphan H II regions thus possibly represent a later evolutionary stage in a gas-stripped tail after the gas supply from part of the disk is exhausted. Then, the once X-ray bright (least dense) gas is pushed to larger tail distances while the dense, star-forming regions decouple from it. At earlier stages, when there is still gas from the disk supplying the tail, the tail has a range of densities, and fast-moving, low-density gas can be spatially coincident with dense lumps. This is probably seen in the narrow tail.

\section{CONCLUSIONS}

We present new APEX ${ }^{12} \mathrm{CO}(2-1)$ observations of ESO 137-001, a Norma cluster spiral galaxy that is currently being violently transformed from a late- to an early-type by strong ram pressure stripping. A prominent $80 \mathrm{kpc} \mathrm{X}$-ray doublestructure tail extends from the galaxy, together with a $40 \mathrm{kpc}$ $\mathrm{H} \alpha$ tail. It also contains warm $\mathrm{H}_{2}$ emission in the inner tail. ESO 137-001 is an excellent example of extreme ram pressure stripping and may become completely gas-free in the near future. Our APEX observations reveal large amounts of cold molecular gas traced by ${ }^{12} \mathrm{CO}(2-1)$ emission in the disk of ESO 137-001 as well as in its gas-stripped tail. It is the first time that a large amount of cold $\mathrm{H}_{2}$ is found in a ram pressure stripped tail. The main results of our analysis are as follows.

1. More than $10^{9} M_{\odot}$ of molecular gas was detected in the three APEX $230 \mathrm{GHz}$ apertures along the tail of ESO 137-001, including a $\sim 40 \mathrm{kpc}$ distant intra-cluster region in its outer part where both X-ray and $\mathrm{H} \alpha$ emission peak. Although the X-factor is uncertain in the special environment of a gas-stripped tail, we assume the Galactic value because the metallicity measured in the tail is close to solar $\left(\sim 0.6 Z_{\odot}\right)$. As suggested by the high warm-tocold (mid-IR to $\mathrm{CO}$ ) molecular gas mass ratio measured in the inner part of the tail, the CO emission may trace not only cold but also warmer molecular gas. Consequently, the standard value of the $\mathrm{X}$-factor could overestimate the $\mathrm{H}_{2}$ content in the tail. Nevertheless, there currently may be more molecular gas in the tail of the galaxy than in its main body.

2. In the most distant $(\sim 40 \mathrm{kpc})$ tail region, more than $10^{8} M_{\odot}$ of $\mathrm{H}_{2}$ was revealed. Since the estimated tidal truncation radius of the galaxy is $15 \mathrm{kpc}$, the detected molecular gas may already be released from the gravitational well of the galaxy. The detected amount is similar to typical molecular masses of tidal dwarf galaxies. We speculate that a ram pressure dwarf galaxy (RPDG) could be forming in this location. More detailed observations measuring the selfgravitation of the object are needed, though.

3. About $1 \times 10^{9} M_{\odot}$ of molecular gas was detected in the APEX aperture centered on the main body of ESO 137-001. This is a factor of at least $\sim 2$ less than the original molecular gas content of the galaxy, as estimated from typical amounts expected in unperturbed spiral galaxies of the same stellar mass.

4. Our observations show that $\mathrm{H}_{2}, \mathrm{H} \alpha$, and X-ray emission can be at observable levels in a single ram-pressure-stripped tail. The amounts of cold and hot gas in the tail are large and similar $\left(\sim 10^{9} M_{\odot}\right)$ and together nearly account for the missing original gas in the disk. Following our measurements, the amount of molecular gas decreases along the tail, while masses of other gas phases (X-ray, ionized) are roughly constant. This could correspond to a decreasing mean gas density along the tail, to long timescales of heating, or to increasing ram pressure that has been able to recently push denser gas to the inner tail regions.

5. SFE was found to be very low in the tail environment $\left(\tau_{\mathrm{dep}, \mathrm{H}_{2}}>10^{10} \mathrm{yr}\right)$, while it is consistent with other spiral galaxies in the main body of ESO 137-001. This indicates that most of the stripped gas does not form stars but remains gaseous and ultimately joins the ICM. Similarly low SFEs are found, for example, in the outer disks of spiral galaxies where $\mathrm{H}$ I is, however, likely to represent most of the ISM and $\mathrm{CO}$ is mostly undetected. This is in contrast to the CObright tail of ESO 137-001, where H I was measured only to upper limits. Star clusters formed in the tail from the gas accelerated by ram pressure to high velocities exceeding the escape speed from the galaxy will contribute to the intra-cluster light population.

6. The ICM thermal (+ ram) pressure at the location of ESO 137-001 in the Norma cluster is similar to midplane gas pressures that occur in the (inner) disks of galaxies. Moreover, the lower limits on the molecular-to-atomic gas ratio in the tail of ESO 137-001 (corresponding to our APEX detections and the ATCA H I upper limits) are consistent with values measured in galactic disks. Nevertheless, the SFE in the tail is much lower than in the galaxies. We suggest that this is due to a low average gas density in the tail, or turbulence driven from interaction with the surrounding ICM, preventing the stripped cold gas from star formation. The elevated ratio of warm-to-cold molecular gas $>0.1$ in the inner tail of ESO 137-001 is close to ratios measured in cool cores of cluster and group central galaxies or in environments where molecular gas is heated by shocks or cosmic rays rather than UV-heated.

7. Our semi-analytic modeling of possible orbits of ESO 137001 in the Norma cluster indicate that the galaxy may be at 
a high velocity of $\sim 3000 \mathrm{~km} \mathrm{~s}^{-1}$ in order to be consistent with a first infall scenario. Consequently, the ram pressure experienced by ESO 137-001 has possibly been strong enough to shift or strip from the disk some gas clumps with column densities exceeding values found in Milky Way typical molecular clouds. Such dense gas in the tail can transform more readily into molecular gas than stripped diffuse gas. Moreover, some fraction of the stripped gas can survive in the molecular phase and contribute to the unprecedented $\mathrm{CO}$ brightness of the gas stripped tail of the galaxy, especially in its inner parts. The molecular gas detected in the outer tail is more likely to originate from in situ transformation of stripped diffuse atomic gas.

Future ALMA observations with high spatial resolution will enable us to study ESO 137-001 in a great detail and to better understand the process of cold gas stripping and mixing with the surrounding ICM, as well as the star formation in the special environment of a ram pressure stripped tail.

We acknowledge support by the Czech Science Foundation grant P209/12/1795, project M100031203 of the Academy of Sciences of the Czech Republic, and the institutional research project RVO:67985815. This research was further supported under Australian Research Council's Discovery Projects funding scheme (project No. 130100664). We thank our anonymous referee for helpful comments that improved the quality of this paper. This research has made use of the NASA/IPAC Extragalactic Database (NED) which is operated by the Jet Propulsion Laboratory, California Institute of Technology, under contract with the National Aeronautics and Space Administration. We further acknowledge the usage of the HyperLeda database (http://leda.univ-lyon1.fr).

Facility: APEX (SHFI)

\section{REFERENCES}

Abramson, A., \& Kenney, J. D. P. 2014, AJ, 147, 63

Abramson, A., Kenney, J. D. P., Crowl, H. H., et al. 2011, AJ, 141, 164

Bigiel, F., Leroy, A., Walter, F., et al. 2010, AJ, 140, 1194

Bigiel, F., Leroy, A., Walter, F., et al. 2008, AJ, 136, 2846

Bigiel, F., Leroy, A. K., Walter, F., et al. 2011, ApJL, 730, L13

Blitz, L., \& Rosolowsky, E. 2004, ApJL, 612, L29

Blitz, L., \& Rosolowsky, E. 2006, ApJ, 650, 933

Boehringer, H., Neumann, D. M., Schindler, S., \& Kraan-Korteweg, R. C. 1996, ApJ, 467, 168

Boissier, S., Boselli, A., Duc, P.-A., et al. 2012, A\&A, 545, A142

Bolatto, A. D., Wolfire, M., \& Leroy, A. K. 2013, ARA\&A, 51, 207

Boselli, A., Cortese, L., Boquien, M., et al. 2014, A\&A, 564, A67

Braine, J., Duc, P.-A., Lisenfeld, U., et al. 2001, A\&A, 378, 51

Butcher, H., \& Oemler, A., Jr. 1978, ApJ, 219, 18

Butcher, H., \& Oemler, A., Jr. 1984, ApJ, 285, 426

Chung, A., van Gorkom, J. H., Kenney, J. D. P., Crowl, H., \& Vollmer, B. 2009, AJ, 138, 1741

Chung, A., van Gorkom, J. H., Kenney, J. D. P., \& Vollmer, B. 2007, ApJL, 659, L115

Cortese, L., Gavazzi, G., Boselli, A., et al. 2006, A\&A, 453, 847

Cortese, L., Marcillac, D., Richard, J., et al. 2007, MNRAS, 376, 157

Courteau, S., Dutton, A. A., van den Bosch, F. C., et al. 2007, ApJ, 671, 203

Crowl, H. H., Kenney, J. D. P., van Gorkom, J. H., \& Vollmer, B. 2005, AJ, 130,65

Dasyra, K. M., Combes, F., Salomé, P., \& Braine, J. 2012, A\&A, 540, A112

Dressler, A. 1980, ApJ, 236, 351

Duc, P.-A., \& Renaud, F. 2013, in Tides in Astronomy and Astrophysics, ed.

J. Souchay, S. Mathis, \& T. Tokieda (Lecture Notes in Physics, Vol. 861; Berlin: Springer), 327

Dumke, M., \& Mac-Auliffe, F. 2010, Proc. SPIE, 7737, 77371J

Ebeling, H., Stephenson, L. N., \& Edge, A. C. 2014, ApJL, 781, L40

Feldmann, R., Gnedin, N. Y., \& Kravtsov, A. V. 2012, ApJ, 758, 127

Finoguenov, A., Briel, U. G., Henry, J. P., et al. 2004, A\&A, 419, 47
Fossati, M., Gavazzi, G., Boselli, A., \& Fumagalli, M. 2012, A\&A, 544, A128 Gavazzi, G., Boselli, A., Mayer, L., et al. 2001, ApJL, 563, L23

Guillard, P., Boulanger, F., Pineau Des Forêts, G., \& Appleton, P. N. 2009, A\&A, 502,515

Guillard, P., Boulanger, F., Pineau des Forêts, G., et al. 2012, ApJ, 749, 158

Gunn, J. E., \& Gott, J. R., III. 1972, ApJ, 176, 1

Hester, J. A., Seibert, M., Neill, J. D., et al. 2010, ApJL, 716, L14

Heyer, M., Krawczyk, C., Duval, J., \& Jackson, J. M. 2009, ApJ, 699, 1092

Huang, S., Haynes, M. P., Giovanelli, R., et al. 2012, AJ, 143, 133

Jáchym, P., Kenney, J. D. P., Ržuička, A., et al. 2013, A\&A, 556, 99

Johnstone, R. M., Hatch, N. A., Ferland, G. J., et al. 2007, MNRAS, 382, 1246

Kapferer, W., Sluka, C., Schindler, S., Ferrari, C., \& Ziegler, B. 2009, A\&A, 499, 87

Kenney, J. D. P., Geha, M., Jáchym, P., et al. 2014, ApJ, 780, 119

Kenney, J. D. P., \& Koopmann, R. A. 1999, AJ, 117, 181

Kenney, J. D. P., Tal, T., Crowl, H. H., Feldmeier, J., \& Jacoby, G. H. 2008, ApJL, 687, L69

Kenney, J. D. P., van Gorkom, J. H., \& Vollmer, B. 2004, AJ, 127, 3361

Kennicutt, R. C., \& Evans, N. J. 2012, ARA\&A, 50, 531

Kroupa, P., \& Weidner, C. 2003, ApJ, 598, 1076

Krumholz, M. R., McKee, C. F., \& Tumlinson, J. 2009, ApJ, 693, 216

Lee, J. C., Gil de Paz, A., Tremonti, C., et al. 2009, ApJ, 706, 599

Lee, J. C., Hwang, H. S., \& Ko, J. 2013, ApJ, 774, 62

Leroy, A. K., Walter, F., Bigiel, F., et al. 2009, AJ, 137, 4670

Leroy, A. K., Walter, F., Brinks, E., et al. 2008, AJ, 136, 2782

Machacek, M., Dosaj, A., Forman, W., et al. 2005, ApJ, 621, 663

Maloney, P. R., Hollenbach, D. J., \& Tielens, A. G. G. M. 1996, ApJ, 466, 561

Murphy, E. J., Kenney, J. D. P., Helou, G., Chung, A., \& Howell, J. H. 2009, ApJ, 694, 1435

Murray, N. 2011, ApJ, 729, 133

Navarro, J. F., Frenk, C. S., \& White, S. D. M. 1996, ApJ, 462, 563

Nesvadba, N. P. H., Boulanger, F., Salomé, P., et al. 2010, A\&A, 521, A65

Nulsen, P. E. J. 1982, MNRAS, 198, 1007

Obreschkow, D., \& Rawlings, S. 2009, MNRAS, 394, 1857

Paturel, G., Petit, C., Prugniel, P., et al. 2003, A\&A, 412, 45

Quilis, V., Moore, B., \& Bower, R. 2000, Sci, 288, 1617

Roediger, E., Brüggen, M., \& Hoeft, M. 2006, MNRAS, 371, 609

Roediger, E., \& Hensler, G. 2005, A\&A, 433, 875

Roussel, H., Helou, G., Hollenbach, D. J., et al. 2007, ApJ, 669, 959

Ruszkowski, M., Bruggen, M., Lee, D., \& Shin, M.-S. 2014, ApJ, 784, 75

Saintonge, A., Kauffmann, G., Kramer, C., et al. 2011, MNRAS, 415, 32

Salomé, P., Combes, F., Revaz, Y., et al. 2011, A\&A, 531, A85

Scott, T. C., Bravo-Alfaro, H., Brinks, E., et al. 2010, MNRAS, 403, 1175

Scott, T. C., Cortese, L., Brinks, E., et al. 2012, MNRAS, 419, L19

Sivanandam, S., Rieke, M. J., \& Rieke, G. H. 2010, ApJ, 717, 147

Smith, R. J., Lucey, J. R., Hammer, D., et al. 2010, MNRAS, 408, 1417

Solomon, P. M., Rivolo, A. R., Barrett, J., \& Yahil, A. 1987, ApJ, 319, 730

Subramanian, K., Shukurov, A., \& Haugen, N. E. L. 2006, MNRAS, 366, 1437

Sun, M., Donahue, M., Roediger, E., et al. 2010, ApJ, 708, 946

Sun, M., Donahue, M., \& Voit, G. M. 2007, ApJ, 671, 190

Sun, M., Jones, C., Forman, W., et al. 2006, ApJL, 637, L81

Sun, M., \& Vikhlinin, A. 2005, ApJ, 621, 718

Tonnesen, S., \& Bryan, G. L. 2008, ApJL, 684, L9

Tonnesen, S., \& Bryan, G. L. 2009, ApJ, 694, 789

Tonnesen, S., \& Bryan, G. L. 2010, ApJ, 709, 1203

Tonnesen, S., \& Bryan, G. L. 2012, MNRAS, 422, 1609

Tonnesen, S., Bryan, G. L., \& Chen, R. 2011, ApJ, 731, 98

van der Wel, A., Bell, E. F., Holden, B. P., Skibba, R. A., \& Rix, H.-W. 2010, ApJ 714, 1779

Vollmer, B., Braine, J., Combes, F., \& Sofue, Y. 2005, A\&A, 441, 473

Vollmer, B., Braine, J., Pappalardo, C., \& Hily-Blant, P. 2008, A\&A, 491, 455

Vollmer, B., Cayatte, V., van Driel, W., et al. 2001, A\&A, 369, 432

Vollmer, B., Wong, O. I., Braine, J., Chung, A., \& Kenney, J. D. P. 2012, A\&A, 543, A33

Wang, Q. D., Owen, F., \& Ledlow, M. 2004, ApJ, 611, 821

Wetzel, A. R. 2011, MNRAS, 412, 49

Wong, O. I., Kenney, J. D. P., Murphy, E. J., \& Helou, G. 2014, ApJ, 783, 109

Wong, T., \& Blitz, L. 2002, ApJ, 569, 157

Woudt, P. A., Kraan-Korteweg, R. C., Lucey, J., Fairall, A. P., \& Moore, S. A. W. 2008, MNRAS, 383, 445

Wyder, T. K., Martin, D. C., Barlow, T. A., et al. 2009, ApJ, 696, 1834

Yagi, M., Gu, L., Fujita, Y., et al. 2013, ApJ, 778, 91

Yagi, M., Komiyama, Y., Yoshida, M., et al. 2007, ApJ, 660, 1209

Yamagami, T., \& Fujita, Y. 2011, PASJ, 63, 1165

Yoshida, M., Ohyama, Y., Iye, M., et al. 2004, AJ, 127, 90

Yoshida, M., Yagi, M., Komiyama, Y., et al. 2008, ApJ, 688, 918

Zu, Y., \& Weinberg, D. H. 2013, MNRAS, 431, 3319 